\title{
Kinematic Artifacts in Prestack Depth Migration
}

\author{
Christiaan C. Stolk* and William W. Symes ${ }^{\dagger}$
}

ABSTRACT
Strong refraction of waves in the migration velocity model introduces kinematic
artifacts - coherent events not corresponding to actual reflectors - into the image vol-
umes produced by prestack depth migration applied to individual data bins. Because
individual bins are migrated independently, the migration has no access to the bin
component of slowness. This loss of slowness information permits events to migrate
along multiple incident-reflected ray pairs, thus introducing spurious coherent events
into the image volume. This pathology occurs for all common binning strategies,
including common source, common offset, and common scattering angle. Since the
artifacts move out with bin parameter, their effect on the final stacked image is min-
imal, provided that the migration velocity model is kinematically correct. However
common image gathers may exhibit energetic primary events with substantial residual
moveout, even with kinematically accurate migration velocity model.

${ }^{*}$ Centre de Mathématiques, Ecole Polytechnique, 91128 Palaiseau Cedex, France, email stolk@math.polytechnique.fr

${ }^{\dagger}$ The Rice Inversion Project, Department of Computational and Applied Mathematics, Rice University, Houston TX 77005-1892 USA, email symes@caam.rice.edu 


\section{INTRODUCTION}

Unstacked migrated image volumes are the raw material of migration velocity analysis. Migration velocities are assessed and estimated by means of the semblance principle: if the velocity is correct, then images formed from different data bins within the unstacked data volume should be the same, at least with respect to phase. Thus image gathers (migrated traces sharing a common surface location), displayed in depth vs. bin parameter, should appear flat. Velocity analysis adjusts migration velocity iteratively to flatten image gathers, the flatness being assessed either directly through visual inspection, or indirectly through velocity spectrum peak location, or both (Yilmaz, 1987; Kleyn, 1983; Claerbout, 1985; Taner and Koehler, 1969).

In principle, any binning scheme can serve as the basis for this type of velocity analysis method, so long as independent images can be formed from each bin - that is, so long as the migration operator can be applied binwise. Common choices are shot and offset binning. Several authors have recently explored formation of image gathers using diffraction sum ("Kirchhoff") operators which image events at selected scattering angles (Xu et al., 2001; Brandsberg-Dahl et al., 1999; De Hoop et al., 2001; Brandsberg-Dahl et al., 2003). We shall refer to this imaging method as common scattering angle Kirchhoff migration.

Strong lateral velocity variation ("complex structure") complicates both the theory and the practice of prestack depth migration. Lateral velocity variation induces multipathing, i.e. the appearance of multiple ray paths and arrival times connecting source and receiver locations with scattering points. Imaging methods successful in models with mild lateral velocity variation may fail in the presence of multipathing. For example, prestack Kirchhoff migration with first arrivals does not always produce accurate (stacked) images in complex structure (Geoltrain and Brac, 1993; Gray and May, 1994). A number of authors have explored Kirchhoff migration with most energetic, as opposed to earliest, 
arrivals (Audebert et al., 1997). The examples presented by Operto et al. (2000) suggest that even most energetic arrivals may not be sufficient to produce high quality images: in some cases, all arrivals carrying significant energy may be required. Recent theoretical work by Ten Kroode et al. (1998) is consistent with this suggestion.

The purpose of this paper is to demonstrate that multipathing generally leads to the presence in unstacked image volumes of spurious coherent events unrelated to actual reflectors, in addition to correctly positioned reflector images. We shall call these spurious events kinematic artifacts, to emphasize that their origin is fundamentally different from that of other coherent image noises, such as diffractions from data truncations and unsuppressed multiple reflections. All common binning strategies, and all binwise prestack migration methods, produce kinematic artifacts in complex structure. As a result, the semblance principle fails for such prestack migration methods in the presence of strong lateral velocity variation: image gathers may not be (entirely) flat, even when the velocity model closely approximates the actual propagation velocity, and all arrivals are used in the migration process.

The failure of the semblance principle is a straightforward consequence of the existence of multiple ray paths connecting reflection points with source and receiver locations. Independent migration of data bins does not give the imaging method access to event slowness in complementary, out-of-bin directions, and therefore permits imaging along several distinct (incident-reflected) ray pairs corresponding to a within-bin event. Thus energy arriving in the data along one ray pair may be imaged at the reflection point of another ray pair, giving rise to a kinematic artifact.

Claerbout (1985) has cited this phenomenon to explain the failure of the exploding reflector model to account properly for all events in zero-offset sections when the velocity model is strongly refracting. In the same way, events in a shot gather determine the 
receiver component of slowness (i.e. rate of change of event arrival time with receiver position), but not the source component, which in principle permits a single event to be imaged along several ray pairs. Nolan and Symes $(1996,1997)$ demonstrated that this could actually occur, using simple synthetic examples. While Nolan and Symes used two-way reverse time finite difference migration to illustrate their conclusions, they apply equally well to Kirchhoff common shot migration using all arrivals, or to common shot migration using depth extrapolation. When the velocity is correct, the artifacts move out with bin parameter, whereas the correct image components do not: this fact explains the disappearance of the artifacts in the final stacked image (Ten Kroode et al., 1998). However imaging artifacts are present with strength equal to (or even sometimes greater than) that of correct image components in the prestack image volume. The correct image components appear flat in the image gathers. However the presence of artifacts renders the image gathers strongly dependent on shot position (non-flat), even with precisely correct velocity.

We will extend the result of Nolan and Symes by demonstrating that kinematic artifacts occur in prestack image volumes produced by common offset and common scattering angle Kirchhoff migration. In particular, contrary to the assertion by Xu et al. (2001), image gathers produced using common scattering angle Kirchhoff migration are not in general flat, even when the velocity is correct. Therefore, Kirchhoff migration in the angle domain does not appear to be categorically superior to Kirchhoff (or other binwise) migration in the offset or shot domains as a basis for velocity analysis.

We will frame our discussion in terms of Kirchhoff migration, because the physics of artifact generation are most evident in the structure of Kirchhoff imaging algorithms and because the version of angle domain imaging which suffers from artifacts is usually formulated as a diffraction sum (and indeed is difficult to formulate in any other way). 
However the reader should be aware that any kinematically equivalent technique will yield the same results. For example, reverse time common shot migration and Kirchhoff common shot migration are equally prone to kinematic artifacts in complex structure. It is image formation from individual bins which is responsible for artifact generation, not any particular method of image computation.

The next section explains the kinematics of multipathing-induced image artifacts, with details spelled out for both common offset and common scattering angle Kirchhoff migration (Prucha et al., 1999; Stolk, 2002). The following section presents two synthetic examples illustrating the predictions of the theory. The first is an acoustic lens model with flat underlying reflector, very similar to that used by Nolan and Symes (1996). This model permits straightforward identification of the ray pairs of both correct reflector images and artifacts. The second example is due to Xu et al. (2001), and consists of a smoothed version of the Marmousi velocity model (Bourgeois et al., 1991) with a flat reflector. Recognition of kinematic artifacts is more difficult in this more complex model, but they are present nonetheless.

We end with a discussion of the implications of our results for velocity analysis. In particular, we contrast the properties of binwise prestack migration established here with those of a prestack migration method not based on independent data bin imaging, namely Claerbout's (1985) "survey sinking" migration. Recent theoretical work by Stolk and De Hoop (2001) has shown that prestack image volumes generated by survey sinking do not exhibit artifacts, and that the focusing characteristic of correct velocity for this type of migration is not affected by strong lateral velocity variation. This comparison suggests some directions for further research.

Two appendices collect mathematical and computational details. 


\section{THE KINEMATICS OF ARTIFACTS}

Given a velocity model, a (potential) reflection point $\mathbf{x}$ is connected to a source position $\mathbf{s}$ by one or more branches of (one-way) traveltime, and similarly to a receiver position $\mathbf{r}$ by one or more branches. The two-way time therefore is indexed on both source and receiver traveltime branches: we write $T^{(i, j)}(\mathbf{x}, \mathbf{s}, \mathbf{r})$ for the two-way time which is the sum of the $i$ th branch from source $\mathbf{s}$ to $\mathbf{x}$ and the $j$ th branch from $\mathbf{x}$ to receiver $\mathbf{r}$.

We do not assume complete or areal coverage, except when otherwise stated, that is, $\mathbf{s}$ and $\mathbf{r}$ do not necessarily densely sample surfaces. Generally these coordinate vectors are themselves functions of other densely sampled parameter vectors. For example, for marine single streamer geometry it is natural to parameterize for $\mathbf{s}$ and $\mathbf{r}$ by source latitude and longitude (source-x and source-y) and source-receiver offset. Source-receiver azimuth, and therefore receiver position, is then a function of $\mathbf{s}$ and $\mathbf{r}$.

Kirchhoff or diffraction sum migration places at an image point $\mathbf{x}$ the sum over sources $\mathbf{s}$, receivers $\mathbf{r}$, and traveltime branches $(i, j)$ of the data $d(\mathbf{r}, \mathbf{s}, t)$ for $t=T^{(i, j)}(\mathbf{x}, \mathbf{s}, \mathbf{r})$, plus possibly some filtering and scaling for amplitudes. When many branches of traveltime exist, the relative phases of the data sums over the branches must be adjusted by application of powers of the Hilbert transform. Also so called true amplitude migration, or migration-inversion, can be produced by properly chosing amplitudes. We have relegated the details of these refinements to Appendix A. For the moment, we simply write for the image amplitude

$$
f_{\text {stack }}(\mathbf{x})=\sum_{(\mathbf{s}, \mathbf{r})} \sum_{(i, j)}(\ldots) d\left(\mathbf{s}, \mathbf{r}, T^{(i, j)}(\mathbf{x}, \mathbf{s}, \mathbf{r})\right)
$$

in which (...) represents amplitudes, filters, and other operators which do not affect the presence or absence of events. The subscript emphasizes that $f_{\text {stack }}(\mathbf{x})$ is the stack of the migrated data volume, which includes contributions from all traces.

An event is characterized by an identifiable arrival time function $T_{\text {data }}(\mathbf{s}, \mathbf{r})$ of source 
position s and receiver position $\mathbf{r}$. We presume throughout our discussion that sampling in $\mathbf{s}$ and $\mathbf{r}$ is sufficiently dense that the source and receiver slownesses of the event

$$
\mathbf{p}_{s}=\nabla_{\mathbf{s}} T, \quad \mathbf{p}_{r}=\nabla_{\mathbf{r}} T
$$

are accurately determined. The event contributes significant image energy at $\mathbf{x}$ if one or more of the diffraction surfaces $t=T^{(i, j)}(\mathbf{x}, \mathbf{s}, \mathbf{r})$ is tangent to the event surface $t=$ $T_{\text {data }}(\mathbf{s}, \mathbf{r})$ for $\mathbf{s}, \mathbf{r}$ within the definition of the event: that is, $f_{\text {stack }}(\mathbf{x})$ gets a contribution from the event if

$$
T^{(i, j)}=T_{\text {data }}, \quad \nabla_{\mathbf{s}} T^{(i, j)}=\mathbf{p}_{s}, \quad \nabla_{\mathbf{r}} T^{(i, j)}=\mathbf{p}_{r} .
$$

Conversely, if a reflector exists at $\mathbf{x}$, then the reflector contributes energy only to events for which equations (2) hold. We will call equations (2) the imaging equations, as they determine the relation between reflector and event locations.

The kinematic artifacts whose existence is the major claim of this paper arise through interaction of two traveltime branches, say indexed by $(i, j)$ and $\left(i^{\prime}, j^{\prime}\right)$, at different image points. Suppose that energy enters the data from a reflector at $\mathbf{x}$ along the incidentreflected ray pair for branch $(i, j)$, i.e. equations (2) hold for $\mathbf{x}, \mathbf{s}, \mathbf{r}, i, j$. Now suppose that equations (2) also hold for another branch of the traveltime, with different indices $\left(i^{\prime}, j^{\prime}\right)$, at another image point $\mathbf{x}^{\prime}$, for the same event, i.e. at the same values of $\mathbf{s}, \mathbf{r}, t, \mathbf{p}_{s}, \mathbf{p}_{r}$. Then the Kirchhoff migration operator (equation (1)) produces image energy at $\mathbf{x}^{\prime}$, whether a reflector actually exists at that location or not. That is, energy arriving in the data due to one ray pair is migrated along another. The result is a kinematic image artifact.

Kinematic artifacts do not occur in general for areal coverage. Idealizing slightly, suppose that $\mathbf{s}$ and $\mathbf{r}$ are coordinates in a plane (or on a line, for imaging based on a 2D Earth model) containing all source and receiver positions, and that $\mathbf{s}$ and $\mathbf{r}$ densely sample regions in this plane. Then $\mathbf{p}_{s}$ is a 2 component vector, representing two of three components of a ray slowness vector. It determines the takeoff velocity vector of the ray 
connecting $\mathbf{s}$ with $\mathbf{x}$, from ray theory, and hence the ray itself. Similarly $\mathbf{p}_{r}$ determines the ray connecting $\mathbf{r}$ with $\mathbf{x}$. So long as these rays intersect at only one point $(\mathbf{x})$ with total time $t=T_{\text {data }}(\mathbf{s}, \mathbf{r})$, no other branch of the traveltime can produce a part of the diffraction surface tangent to the event surface at $\left(\mathbf{s}, \mathbf{r}, T_{\text {data }}(\mathbf{s}, \mathbf{r})\right)$, so necessarily $i^{\prime}=i, j^{\prime}=j$, and $\mathbf{x}^{\prime}=\mathbf{x}$. The condition that the total time determine the reflection point is the Traveltime Injectivity Condition ("TIC") of Ten Kroode et al. (1998), who gave a rigorous proof that artifacts do not exist in the stacked image for areal coverage (or linear coverage, for 2D) provided that TIC is assumed. While it is possible to construct velocity models and areal coverage geometries that violate the TIC, it is satisfied in many common imaging situations, for instance if incident and reflected rays do not turn horizontal. We will tacitly assume that TIC applies to the imaging problem with areal source and receiver coverage throughout our discussion.

When coverage is not areal and multipathing occurs, kinematic artifacts are the rule, rather than the exception. The example that concerns us here is the imaging of an individual data bin. We will treat two cases explicitly, both in 2D, which will be illustrated in the next section. In both cases 3D generalizations are clear; generation of artifacts is if anything easier for the analogous 3D imaging situations.

In both cases $\mathbf{s}$ and $\mathbf{r}$ are positions on a line, represented by scalar coordinates $s$ and $r$. Migration of the common offset bin with half-offset $h=(r-s) / 2$ is given by

$$
f_{\text {offset }}(\mathbf{x}, h)=\sum_{s} \sum_{(i, j)}(\ldots) d\left(s, s+2 h, T^{(i, j)}(\mathbf{x}, s, s+2 h)\right) .
$$

An event in an offset bin determines only the (scalar) midpoint slowness $p_{m}=p_{r}+p_{s}$; it does not determine the offset slowness $p_{h}=p_{r}-p_{s}$, as the offset moveout is not available. Therefore the correct imaging equations for a single offset bin are

$$
T^{(i, j)}=T_{\text {data }}, \quad \frac{\partial T^{(i, j)}}{\partial s}+\frac{\partial T^{(i, j)}}{\partial r}=\frac{\partial T^{(i, j)}}{\partial m}=p_{m}
$$


were $m$ is the midpoint $m=(s+r) / 2$.

Compared to equations (2), equations (4) have lost an entire equation: the offset component $p_{h}$ of event moveout does not constrain the reflection point. So it is perhaps not surprising equations (4) have in general many more solutions. In particular, multipathing generally induces kinematic artifacts in offset image bins, as described above. The paper by Prucha et al. (1999) gave a simple example of an artifact at zero offset, while Stolk (2002) showed that such artifacts exist at all offsets, also at the purely kinematic level (map migration). The next section shows explicit Kirchhoff migrations corresponding to the kinematics of Stolk (2002).

If we sum the right hand side of equation (3) over half offset $h$, we obtain the right hand side of equation (1) (2D version). Since the stacked image has no artifacts, as we have argued, stacking must remove those present in the offset bins - that is, the artifacts must move out within the offset CIGs, whereas the correct image components do not. The examples of the next section illustrate this property of kinematic artifacts.

The second example, common scattering angle migration, is a bit more involved. Reflector dip $\nu$ and scattering (opening) angle $\theta$ determine (in 2D) an incident - reflected ray pair (see Figure 1. In this figure $\beta$ and $\alpha$ are the takeoff angles for the source and receiver rays at the reflection point). Thus we can view $s$ and $r$, as well as $t$, as functions of $\mathbf{x}, \theta$, and $\nu$. The common scattering angle Kirchhoff migration operator is

$$
f_{\text {angle }}(\mathbf{x}, \theta)=\sum_{\nu}(\ldots) d(s(\mathbf{x}, \theta, \nu), r(\mathbf{x}, \theta, \nu), T(\mathbf{x}, \theta, \nu))
$$

(see Xu et al. (2001) and Brandsberg-Dahl et al. (2003)). Like equation (3) the expression for $f_{\text {angle }}(\mathbf{x}, \theta)$ is essentially a summation of data along a curve (in $\left.2 \mathrm{D}\right)$ in the $(s, r, t)$ space. Note that since $\mathbf{x}, \theta, \nu$ determine the scattering ray pair completely, the summation over traveltime branches is already implicit in the dependence on these variables, so that we do not need to supply the traveltime function with branch indices. 
It is important to understand that the imaging method (5) which we call common scattering angle Kirchhoff migration, is not equivalent, even kinematically, to the "wave equation angle domain migration" described by Prucha et al. (1999) and Sava et al. (2001). We will have a bit more to say about the latter algorithm in the concluding section.

It is not so easy to envision the operator defined in equation (5) as defining the restriction of the operator defined in equation (1) to a data bin, but in fact that is precisely its identity. The bin must be described in the phase space of the data: it consists of those event elements which are tangent to the constant scattering angle diffraction curve (surface in 3D), parameterized by $\nu$.

The imaging equations which express this tangency are

$$
T(\mathbf{x}, \theta, \nu)=T_{\text {data }}(s(\mathbf{x}, \theta, \nu), r(\mathbf{x}, \theta, \nu)), \quad \frac{\partial T}{\partial \nu}(\mathbf{x}, \theta, \nu)=p_{s} \frac{\partial s}{\partial \nu}(\mathbf{x}, \theta, \nu)+p_{r} \frac{\partial r}{\partial \nu}(\mathbf{x}, \theta, \nu)
$$

Once again, the system (6) has one fewer equation than does the system (2), so it is not surprising that it has many more solutions in general.

Appendix A describes a general formulation of binwise Kirchhoff imaging, which treats all binning schemes, including the two just described, in a unified way.

\section{EXAMPLES}

In this section we present two 2D examples which illustrate the formation of kinematic artifacts in common offset and common scattering angle prestack migration. The first model contains a horizontal reflector below a low velocity lens, and closely resembles the model used by Nolan and Symes (1996), also Prucha et al. (1999). The lens is sufficiently

refracting that multipathing occurs. The second example is based on a slight smoothing of the Marmousi velocty model (Bourgeois et al., 1991), which is well known to produce multipathing. Reflectivity is again supplied by a flat reflector, at the level of the "target" 
structure in the original Marmousi model. This "Marmousi plus flat reflector" model was introduced by Xu et al. (2001).

We demonstrate the presence of image artifacts in two ways. By solving the imaging equations (i.e. equations (4) or (6)) we determine rays that lead to artifacts and predict the positions of both correctly and incorrectly located reflectors in (offset and angle) bin images and common image point gathers. Second we migrate synthetic data using the numerical implementation of Kirchhoff prestack depth migration described in Appendix A. By combining the results we can clearly distinguish between kinematic artifacts and other sources of "noise" that may be present in the image. The results show that artifacts are present and are energetically of the same order of magnitude as the correctly positioned reflectors. There is strong agreement between kinematic predictions of reflector locations and apparent reflectors in the migrated image volume.

\section{The Lens Model}

The first velocity model contains a low velocity Gaussian lens centered $1 \mathrm{~km}$ below the origin of the coordinate system (on the recording surface $z=0$ ). The velocity is given explicitly by

$$
c(x, z)=1-0.4 e^{-9\left(x^{2}+(z-1)^{2}\right)}
$$

in $\mathrm{km} / \mathrm{s}$. We locate the horizontal reflector at depth $z=2 \mathrm{~km}$. Velocity model and reflector are displayed in Figure 2. Some of the rays and wave fronts produced by this model are displayed in Figure 3. A triplication occurs below the lens, leading to three branches of one-way traveltime, numbered 1 to 3 as indicated in the figure (cf. Appendix A, section on migration operators).

We generated a synthetic dataset for this model using a finite difference wave equation solver of order 2 in time and 4 in space. To simulate a reflector, we introduce a jump in the velocity profile, by setting the velocity equal to $1.15 \mathrm{~km} / \mathrm{s}$ below $z=2 \mathrm{~km}$, i.e. an 
increase of about $15 \%$. The geometry is such that no multiple reflections are recorded. The sampling was adequate in all directions (ten gridpoints per wavelength), and the time sample rate high enough, to yield at most $2 \%$ error in phase velocity and no visible grid dispersion (Levander, 1989). The source pulse was a $\delta$-function, bandlimited by a $[4,10$, 20, 40] Hz zero phase bandpass filter. The data consists of 401 shot records for $s$ ranging from -2 to $2 \mathrm{~km}$ with shot spacing $0.01 \mathrm{~km}$. For each shot the response was recorded at 401 receivers with the same coverage and sample rate as the shots. The recorded data simply sampled the simulated pressure field at a depth of $10 \mathrm{~m}$ below the surface.

No absorbing boundary conditions were applied, but the domain was chosen so large that reflections from the boundary arrived significantly later than reflections from the horizontal reflector, with different moveout, so that boundary reflections have no significant effect on the migrated image volume. Figure 4 displays a shot record for $s=-0.5$ $\mathrm{km}$.

A numerical implementation of Kirchhoff prestack depth migration for the offset and angle domains (equations (3) and (5) respectively) produced offset- and angle-dependent image volumes $f_{\text {offset }}(\mathbf{x}, h)$ and $f_{\text {angle }}(\mathbf{x}, \theta)$ respectively. The stacked image $\left(f_{\text {stack }}(\mathbf{x})\right.$, equation (1)) amounts to the summation of $f_{\text {offset }}(\mathbf{x}, h)$ over $h$, or $f_{\text {angle }}(\mathbf{x}, \theta)$ over $\theta$; except for discretization effects, the results should be the same. Figure 5 shows the stacked image: the reflector is indeed recovered at the correct position, and no artifacts appear, as predicted by Ten Kroode et al. (1998).

The numerical implementation of Kirchhoff migration used in these examples is described in Appendix A.

\section{Common offset migration}

To determine the position and slownesses of an event in the data, we trace source and receiver rays from the reflection point in the subsurface, determine where they 
intersect the acquisition plane, given in this example by $z=0.01 \mathrm{~km}$, and calculate $s, r, t=T_{\text {data }}(s, r), p_{s}$, and $p_{r}$. The complete set of reflection events, correct and artifact, corresponding to this event are obtained by solving the imaging equations (4).

Solving these equations involves a combination of ray tracing and root finding. Appendix B describes the solution method in detail.

Figure 6 displays the results of this analysis for a reflecting element at $\mathbf{x}=(0.303,2)$ $\mathrm{km}$, with opening angle 28.05 degrees (chosen so that the offset was equal to $1 \mathrm{~km}$ ). Migration forms an image at three positions, corresponding to the three ray pairs shown in the figure. This prediction is completely kinematic; to see whether significant energy is imaged at these positions we overlay the constant offset image for offset $h=1 \mathrm{~km}$, with

the ray pairs in Figure 7. Indeed roughly equivalent image amplitude occurs at all three positions, with apparent reflector orientation as predicted by the kinematic computation.

We have similarly analyzed the kinematics of offset common image gathers ("CIGs"), and thus predicted the kinematics of events in the offset CIG for $x=0.3 \mathrm{~km}$. These kinematic predictions are the colored lines in Figure 8, the different colors corresponding to different combinations of traveltime functions (i.e. index pairs $(i, j)$ ). The kinematic predictions are overlain on the gray scale plot of the CIG produced by prestack depth migration. All kinematic predictions coincide with events in the migrated data.

\section{Common scattering angle migration}

Kinematic predictions of image components, both correct and artifact, follow from an analysis similar to that used in the common offset case. Algorithmic details are explained in Appendix B.

Figure 9 shows the ray pairs corresponding to correct image and artifacts for data due to reflection at $\mathbf{x}=(0.3,2) \mathrm{km}$, opening angle 40 degrees and for reflection at $\mathbf{x}=(0,2)$ 
$\mathrm{km}$ (below the lens) opening angle 10 degrees. In addition to the ray pairs, we display the isochrons (the solutions in position $\mathbf{x}$ of $t=T^{(i, j)}(\mathbf{x}, r, s)$ for the various branches $(i, j)$ ). The various images of a given event, correct and artifact, occur at different opening angles, therefore this picture cannot be compared with a single constant angle image. A constant angle image for angle 30 degrees is given in Figure 10.

Figure 11 shows a gray scale plot of the angle CIG for $x=0.3 \mathrm{~km}$, with the predicted events superimposed (colored lines). Again all the predicted artifacts can be observed in the synthetic data example and there is strong agreement between the kinematic and synthetic data plots. Angle CIG's for $x=0 \mathrm{~km}$ and $x=0.6 \mathrm{~km}$ are given in Figure 12 . Again artifacts are present and have the same order of magnitude as the correct image. As can be expected the artifact moves to larger angles for increasing $x$.

\section{Example based on the Marmousi model}

To establish the presence of artifacts in geologically more realistic models, we examine the "canonical test case" model presented by Xu et al. (2001). This model is based on a smoothed version of the Marmousi velocity model (Bourgeois et al., 1991), which offers considerable lateral heterogeneity (modeling the results of salt tectonics and faulting) and consequent multipathing. Smoothing removes most of the passband reflectivity of the original model; in its place, the "canonical test case" positions a flat reflecting layer at depth. The presence of only two flat reflectors permits the identification of ray pairs related to coherent events in the image volume, which would be extremely difficult, if not impossible, with a typically dense distribution of reflectivity (as is present in the Marmousi model itself). This "canonical test case" model is therefore very well suited

for evaluating the effect of multipathing on the output of imaging algorithms with the sort and degree of lateral heterogeneity that might be provided by complex geological structure. 
Despite the presence of only two reflectors, the very complex ray geometry of this model renders explicit identification of reflection kinematics quite difficult. For this reason, and because there has been some speculation that common scattering angle Kirchhoff migration would be immune from kinematic artifacts (Xu et al., 2001), we will examine only the effect of multipathing on common scattering angle image gathers. As illustrated in the discussion of the lens model example, common offset and common shot image gathers could be treated in the same way.

The data approximate Born scattering for a low-contrast flat reflector: they are the difference between data for a smooth reference model and a perturbed model. The smooth reference model is a smoothed version of the Marmousi model, low-pass filtered by a Gaussian kernel of half-power radius $150 \mathrm{~m}$. This degree of smoothing has been described as the maximum permissible for accurate migration/inversion of the Marmousi dataset (Versteeg, 1993). The perturbed model was the same smoothed Marmousi model, but now with $10 \mathrm{~m} / \mathrm{s}$ added to the velocity at depths between $2400 \mathrm{~m}$ and $2500 \mathrm{~m}$. The model with the position of the perturbation layer indicated is displayed in Figure 13.

The models were discretized on a $5 \mathrm{~m} \times 5 \mathrm{~m}$ grid, and the corresponding seismic survey simulated using a finite difference method. Acquistion geometry closely resembled that of the original survey (Bourgeois et al., 1991), with 240 sources spaced $25 \mathrm{~m}$ apart ranging from 3000 to $8975 \mathrm{~m}$ from the west end of the model, 96 point receivers spaced $25 \mathrm{~m}$ apart, with offsets from $-100 \mathrm{~m}$ to $-2475 \mathrm{~m}$ (these offsets differed from those in the original synthetic survey distributed by IFP, where the first offset was at $-200 \mathrm{~m}$ and the last at $-2575 \mathrm{~m}$ ). Recording time was 3 seconds, sampled at $4 \mathrm{~ms}$. Source and receiver depths were $25 \mathrm{~m}$ (these depths also differed from those in the original data). The source was isotropic and dilatational. The source pulse was a $\delta$-function bandlimited by a $[5,13,40,55] \mathrm{Hz}$ bandpass filter (again differing from that used in the original data) 
with a time delay of $56 \mathrm{~ms}$. Figure 14 displays a typical shot gather.

Multipathing is particularly prevalent in the right part of the model. We will therefore study an angle common image gather for horizontal position $x=6.2 \mathrm{~km}$, see Figure 17 . A version of this image gather is also displayed as Figure 14(b) in (Xu et al., 2001). The two figures are quite similar in strength and position of events. Minor apparent amplitude and wavelet differences are presumably due to implementation differences between the two migration codes, and to differences in display parameters.

To produce this image gather, we shot ray fans from a range of points along the vertical line $x=6.2 \mathrm{~km}$. One such ray fan is shown in Figure 15 (in fact a larger number of rays was used to obtain sufficient coverage at the surface). As in Appendix B, we denote by $s(x, z, \beta)$ the source coordinate as a function of subsurface point and angle. Each branch of traveltime corresponds to an interval of angles $\beta$ where $\frac{\partial s}{\partial \beta}$ is either positive or negative (but does not change sign). One-way traveltime branches were identified in the $(z, \beta)$ plane according to Figure 16, i.e. along a vertical line through $x=6.2 \mathrm{~km}$. For each of the identified branches we computed traveltime, arrival angle at the surface, and an estimate of the amplitude factor $\left(\frac{\partial s}{\partial \beta}\right)^{-1 / 2}$ (which is the dominant factor in the amplitude, see (Xu et al., 2001)). These were then used to evaluate the two-way traveltime branches and other components of the common scattering angle Kirchhoff sum (equation 5), which outputs the image gather as a function of depth and scattering angle.

Figure 17 clearly displays nonhorizontal events of significant energy. The same events are also identifiable in Figure 14(b) of (Xu et al., 2001). To establish the kinematic nature of these events, we focus on the large event at depth 2100 to 2200, dipping down to the right, surrounded by the box in Figure 17. By computing separately the image for each branch it is seen that this event comes from the combination of branches $(10,2)$ (cf. Figure 16). This is shown in the image in Figure 18, where this combination of branches 
is omitted, and the event is absent.

Once again we solve the imaging equations, angle version (the system (6)) using the method explained in Appendix B. The solution is a great deal more laborious than was the case for the lens model example, due to the number and irregularity of the traveltime branches. In this way we found that the event responsible for the artifact came from reflections between $x=6.6 \mathrm{~km}$ and $x=6.7 \mathrm{~km}$ on the reflector. The position of the artifact as computed from the traveltimes is displayed in Figure 19. Some of the rays corresponding to artifacts are given in Figure 20.

\section{DISCUSSION}

The preceding examples establish that kinematic artifacts, i.e. coherent events not corresponding to actual reflectors, can exist in prestack image volumes produced by binwise migration, given strong lateral velocity variation and concommitant multipathing. Such artifacts can appear in the output of both common offset and common angle Kirchhoff prestack depth migration, and we have given explicit, clear-cut 2D examples of this phenomenon. In particular, formulation of Kirchhoff migration in the common angle domain does not suppress imaging artifacts, contrary to assertions in the literature of Xu et al. (2001).

We have focused our attention on Kirchhoff migration, but in fact the same conclusions hold for any binwise migration method including contributions from all arrivals, such as reverse time or depth extrapolation common shot migration. For example, Nolan and Symes $(1996,1997)$ have exhibited kinematic artifacts in reverse time common shot migration image volumes. The examples by Operto et al. (2000) illustrate the necessity of including all arrivals carrying significant energy to maintain image quality in the presence of multipathing; the relevant theory also suggests this requirement (Ten Kroode et al., 
1998; Nolan and Symes, 1997). However it is precisely the interaction of various arrival branches that creates kinematic artifacts.

Perhaps the most striking conclusion to be drawn from our analysis is that image gathers produced by binwise migration are not in general flat in the presence of strong refraction, even when the velocity model is precisely correct, because of the production of kinematic artifacts. Flattening image gathers is the underlying mechanism of practical velocity analysis. Gathers may be contaminated by many types of coherent noise. However kinematic artifacts can move out in a wide variety of styles, as even our simple examples illustrate, and may be particularly difficult to distinguish from events which should be flattened by velocity updating. For a nice example of this possible confusion, see Nolan and Symes $(1996,1997)$. Artifacts bear no particular space-time relation to the actual reflectors which they spoof: unlike multiple reflections, they can appear either earlier or later than the source reflector in an image gather, and are more or less equally energetic. For all of these reasons, processing designed to suppress multiple reflection energy or edge effects will not attenuate kinematic artifacts, which will be present whenever primary reflections are imaged. Thus the presence of kinematic artifacts will complicate migration velocity analysis in complex structure. The complication could easily be fatal for automated velocity analysis algorithms, such as differential semblance optimization (Gockenbach and Symes, 1999; Mulder and ten Kroode, 2002).

It might be objected that most migration velocity modeling does not include features of the size of the Gaussian anomaly of our first example (0.25 km half-power radius), and therefore our examples exhibit multipathing more severe than would be generated by the typical migration velocity model. However, note that the paper by Brandsberg-Dahl et al. (2003) also exhibits kinematic artifacts in common scattering angle Kirchhoff migration of model data. While these authors do not present a full ray-based analysis, as we do 
here, there is little room for doubt that they describe precisely the phenomenon explained in the present work. The two synthetic models used by Brandsberg-Dahl et al. (2003) are a "cartoon" gas cloud model, containing a Gaussian lens of size and strength very similar to that used in our lens model, and a geologically derived model of a part of the Valhall field. The Valhall model also contains low-compressional-velocity lenses, of similar size and strength to those used in our lens model, but of course less regular in geometry. We note that lateral velocity contrasts of $50 \%-100 \%$ are also found near the edges of salt and chalk bodies. Such velocity contrasts must also produce pronounced multipathing (amongst other pathologies) similar to that produced by the lens model. These examples suggest that our lens model may not be so extreme after all.

Moreover, we have also exhibited the generation of kinematic artifacts for Kirchhoff common scattering angle migration in the smoothed Marmousi velocity model. The structures in the Marmousi model responsible for its strongly refractive nature (the fault blocks in the center of the model) have diameters on the order of $1 \mathrm{~km}$ or larger, and features of this size are certainly accessible to migration velocity analysis (Liu and Bleistein, 1995). Smoothing on scales like $150 \mathrm{~m}$ is commonly applied to velocity models to stabilize ray tracing. Also, evidence exists that more drastic smoothing prevents accurate imaging of the Marmousi reflectivity structure (Versteeg, 1993). Insofar as the Marmousi model is representative of complex geologic structure, multipathing of the severity discussed here is expected to occur in the Earth, and migration velocity analysis will have to take it into account to image such structure successfully.

Clearly a field data example illustrating the appearance of kinematic artifacts would be most interesting. The theory and examples presented here and elsewhere leave little doubt that artifacts occur in prestack image volumes near and under gas chimneys, salt flanks, and other highly refracting structures. Indeed, Brandsberg-Dahl et al. (2003) show 
angle CIGs from Kirchhoff-migrated field data (Valhall OBS) in which kinematic artifacts appear to be present. However the purpose of examples in the context of this paper is to verify the appearance in migrated volumes of image components predicted by the theory. Illustration of our key contention - that image gathers are not flat for "correct" velocity due to multiple images of primary reflections - requires that both a "correct" velocity model and primary reflection energy be identified first. This is a nontrivial task precisely in those situations of strong lateral velocity variation that give rise to the phenomenon under study. Therefore we have limited ourselves in this exposition to synthetic examples, where the "correct" velocity is under our control.

Given the blanket nature of our result - that all of the common binning schemes suffer from kinematic artifact production - it is remarkable that an alternative exists. Very recently, evidence has emerged that "survey-sinking" or "double square root" migration, introduced by Claerbout (1985) does not generate kinematic artifacts (Stolk and De Hoop, 2001). The same is true for the wave equation common angle migration techniques (Prucha et al., 1999; Sava et al., 2001), which are variants on survey-sinking. While these algorithms were introduced as applications of wavefield depth extrapolation, the fundamental reason for the absence of artifacts has nothing to do with depth extrapolation per se. The essential feature of these algorithms, responsible for the absence of kinematic artifacts, is that they do not migrate individual image bins: each output point in the prestack image volume depends (in principle) on the entire data volume, in such a way that migration of energy along the wrong ray pair is simply impossible.

The difference is perhaps best explained by comparing prestack Kirchhoff common offset migration (equation (3)) with a diffraction sum expression for survey-sinking migration. [This comparison also emphasizes our point that depth extrapolation is not the algorithm feature which guarantees artifact avoidance.] Survey sinking introduces a (vec- 
tor) parameter of the image volume, generally called (half) offset and denoted by $\mathbf{h}$. The name is natural in view of Claerbout's (1985) motivation of survey sinking but in fact this parameter cannot be identified with the source-receiver offset of the survey geometry - for example, it does not need to be horizontally oriented (Biondi and Shan, 2002), though the original definition of Claerbout (1985), standard practice, and the results of Stolk and De Hoop (2001) all make that assumption. Introduce the survey sinking two way time branches $T^{(i, j)}(\mathbf{x}, \mathbf{h}, \mathbf{s}, \mathbf{r})$ - the sum of the $i$ th branch of the one-way time from $\mathbf{s}$ to $\mathbf{x}-\mathbf{h}$ and the $j$ th branch of the one-way time from $\mathbf{x}+\mathbf{h}$ to $\mathbf{r}$. Then the Kirchhoff representation of survey-sinking migration is

$$
f_{\mathrm{ss}}(\mathbf{x}, \mathbf{h})=\sum_{\mathbf{s}, \mathbf{r}} \sum_{i, j}(\ldots) d\left(\mathbf{s}, \mathbf{r}, T^{(i, j)}(\mathbf{x}, \mathbf{h}, \mathbf{s}, \mathbf{r})\right)
$$

Claerbout's imaging condition is: extract the zero-offset section. Clearly at $\mathbf{h}=\mathbf{0}$ equation (8) becomes identical to equation (1) so that $f_{\mathrm{ss}}(\mathbf{x}, 0)=f_{\text {stack }}(\mathbf{x})$. The nonzero offset output also involves the entire data set: this is not a binwise migration algorithm, and the algorithm accesses full events for each $\mathbf{h}$, unrestricted by data binning. Therefore full slowness information is available at each $\mathbf{h}$.

The paper by Stolk and De Hoop (2001) contains a demonstration that equation (8) actually yields the same migrated image as survey sinking via the double square root equation as defined in Claerbout (1985), and also that this form of prestack migration produces an image volume uncontaminated by kinematic artifacts whenever the same is true of the stacked image. That is: when the migration velocity is kinematically accurate, the output of survey sinking migration is concentrated or focused at zero offset. The result of wave equation angle domain migration is essentially survey sinking migration followed by a Radon transform in offset and time (Prucha et al., 1999) or depth (Sava et al., 2001), so these methods are also artifact-free, i.e. produce image gathers flat in angle. It has been suggested that the focusing property of survey sinking migration could be used as 
the basis of a velocity analysis method (Schultz and Sherwood, 1982; Claerbout, 1985). Indeed the focusing analysis method of Faye and Jeannot (1986) is one implementation of this idea. Our results suggest that velocity analysis based on survey-sinking migration and its conceptual relatives may be particularly appropriate for accurate imaging in complex structure.

\section{ACKNOWLEDGEMENTS}

We are grateful to Gilles Lambaré for providing the Marmousi-based data. The authors thank Biondo Biondi for many useful conversations. We thank the Associate Editor and referee William Schneider for very helpful comments and criticisms which resulted in a much improved presentation. The work reported here was partly supported by The Rice Inversion Project. The first author also acknowledges the support of the Mathematical Sciences Research Institute, through NSF grant DMS-9810361. 


\section{REFERENCES}

Audebert, F., Nichols, D., Rekdal, T., Biondi, B., Lumley, D., and Urdaneta, H., 1997, Imaging complex geologic structure with single arrival Kirchhoff prestack depth migration: Geophysics, 62, 1533-1543.

Biondi, B., and Shan, G., 2002, Prestack imaging of overturned reflections by reverse time migration: 72nd Ann. Internat. Mtg., Soc. Expl. Geophys., Expanded Abstracts, $1284-1287$.

Bourgeois, A., Lailly, P., and Versteeg, R., 1991, The Marmousi model in Versteeg, R., and Grau, G., Eds., The Marmousi Experience: IFP/Technip.

Brandsberg-Dahl, S., De Hoop, M., and Ursin, B., 1999, Velocity analysis in the common scattering-angle/azimuth domain: 69th Ann. Internat. Mtg., Soc. Expl. Geophys., Expanded Abstracts, 1715-1718.

Brandsberg-Dahl, S., de Hoop, M. V., and Ursin, B., 2003, Focusing in dip and AVA compensation on scattering-angle/azimuth common image gathers: Geophysics, 68, $232-254$.

Burridge, R., and Beylkin, G., 1988, On double integrals over spheres: Inverse Problems, 4, $1-10$.

Claerbout, J., 1985, Imaging the earth's interior: Blackwell Scientific Publishers, Oxford.

De Hoop, M. V., Symes, W. W., Brandsberg-Dahl, S., and Ursin, B., 2001, Tomographic migration velocity analysis by differential semblance optimization with respect to scattering-angle/azimuth in anisotropic elastic media: Technical Report CWP-374, Center for Wave Phenomena, Colorado School of Mines.

Faye, J.-P., and Jeannot, J.-P., 1986, Prestack migration velocities from focusing depth analysis: 56th Ann. Internat. Mtg., Soc. Expl. Geophys., Expanded Abstracts, 438-440.

Geoltrain, S., and Brac, J., 1993, Can we image complex structures with first-arrival traveltime?: Geophysics, 58, 564-575. 
Gockenbach, M., and Symes, W., 1999, Coherent noise suppression in velocity inversion: 69th Ann. Internat. Mtg., Soc. Expl. Geophys., Expanded Abstracts, 1719-1722.

Gray, S., and May, W., 1994, Kirchhoff migration using eikonal equation traveltimes: Geophysics, 59, 810-817.

Kleyn, A., 1983, Seismic reflection interpretation: Applied Science Publishers, New York.

Levander, A., 1989, Finite difference forward modeling in seismology in James, D. E., Ed., The Encyclopedia of Solid Earth Geophysics:: Van Nostrand Reinhold, 410-431.

Liu, Z., and Bleistein, N., 1995, Mathematical analysis of residual moveout and velocity analysis: 65th Ann. Internat. Mtg., Soc. Expl. Geophys., Expanded Abstracts, 12011204.

Mulder, W., and ten Kroode, A., 2002, Automatic velocity analysis by differential semblance optimization: Geophysics, 67, 1184-1191.

Nolan, C. J., and Symes, W. W., 1996, Imaging and coherency in complex structure: 66th Ann. Internat. Mtg., Soc. Expl. Geophys., Expanded Abstracts, 359-363.

Nolan, C., and Symes, W., 1997, Global solution of a linearized inverse problem for the wave equation: Comm. P. D. E., 22, 919-952.

Operto, M. S., Xu, S., and Lambaré, G., 2000, Can we quantitatively image complex structures with rays?: Geophysics, 65, 1223-1238.

Prucha, M., Biondi, B., and Symes, W., 1999, Angle-domain common image gathers by wave-equation migration: 69th Ann. Internat. Mtg., Soc. Expl. Geophys., Expanded Abstracts, 824-827.

Sava, P., Biondi, B., and Fomel, S., 2001, Amplitude preserved common image gathers by wave equation migration: 71st Ann. Internat. Mtg., Soc. Expl. Geophys., Expanded Abstracts, 296-299.

Schultz, P., and Sherwood, J., 1982, Depth migration before stack: Geophysics, 45, 376393. 
Stolk, C. C., and De Hoop, M. V., 2001, Seismic inverse scattering in the 'waveequation' approach: Preprint 2001-047, The Mathematical Sciences Research Institute, http://msri.org/publications/preprints/2001.html.

Stolk, C. C., 2000, Microlocal analysis of a seismic linearized inverse problem: Wave Motion, 32, 267-290.

Stolk, C. C., 2002, Microlocal analysis of the scattering angle transform: Comm. P. D. E., 27, 1879-1900.

Taner, M., and Koehler, F., 1969, Velocity spectra: digital computer derivation and application of velocity functions: Geophysics, 34, 859-881.

Ten Kroode, A. P. E., Smit, D. J., and Verdel, A. R., 1998, A microlocal analysis of migration: Wave Motion, 28, 149-172.

Versteeg, R., 1993, Sensitivity of prestack depth migration to the velocity model: Geophysics, 58, 873-882.

Xu, S., Chauris, H., Lambaré, G., and Noble, M., 2001, Common angle migration: A strategy for imaging complex media: Geophysics, 66, 1877-1894.

Yilmaz, O., 1987, Seismic data processing: Soc. Expl. Geophys.

\section{APPENDIX A \\ A GENERAL FORMULATION OF PRESTACK KIRCHHOFF MIGRATION AND THE IMAGING EQUATIONS}

In this appendix we present a unified theory of prestack Kirchhoff migration, applicable to all domains (shot, offset, angle,...). This formulation facilitates a uniform analysis of artifact generation, via conditions which we call the imaging equations. We also describe the particular choices of discretization used in our computational implementation of Kirchhoff migration. 


\section{Migration Operators}

A reflecting pair of geometrical optics rays can be parameterized by the reflection point $\mathbf{x}$ in the subsurface and the takeoff angles $(\beta, \alpha)$ for the source and receiver rays. For 2D modeling, $\mathbf{x}=(x, z), z$ denoting depth, and $\beta, \alpha$ are ordinary angles. For 3D modeling, $\mathbf{x}=(x, y, z)$, and $\beta, \alpha$ are solid angles.

We assume for convenience that the source and receiver positions are located on a constant-depth acquisition plane $z=0$. The analysis remains valid if these positions lie on non-planar surfaces instead, and if sources and receivers lie at different depths. The data $(\mathbf{x}, \beta, \alpha)$ determine the intersection point $\mathbf{s}(\mathbf{x}, \beta)$ of the source ray with the acquistion plane together with the corresponding traveltime $\mathbf{t}_{\mathbf{s}}(\mathbf{x}, \beta)$, and similarly for the receiver $\mathbf{r}(\mathbf{x}, \alpha), \mathbf{t}_{\mathrm{r}}(\mathbf{x}, \alpha)$, see Figure 1.

The map $(\mathbf{x}, \alpha) \mapsto(\mathbf{x}, \mathbf{s}(\mathbf{x}, \alpha))$ in general maps several angles $\alpha$ to the same source (multipathing). Therefore there are multiple inverse functions mapping $(\mathbf{x}, \mathbf{s})$ to the takeoff angle, which we will denote by $\alpha^{(i)}(\mathbf{x}, \mathbf{s})$. Each inverse function is defined for $(\mathbf{x}, \mathbf{s})$ in a certain set that we will denote by $D^{(i)}$, and corresponds to a branch one-way traveltime, denoted $T_{1}^{(i)}(\mathbf{x}, \mathbf{s})$, defined for $(\mathbf{x}, \mathbf{s}) \in D^{(i)}$.

Since the traveltime branches are generally only local, not global, it will be necessary to cut off or mute the data to be summed over. The data must be multiplied by a function that smoothly goes to zero near the boundary of the summation domain (a

tapering function). Therefore we define a smooth cutoff (mute) function $A_{1}^{(i)}(\mathbf{x}, \mathbf{s})$ on $D^{(i)}$, identically equal to one sufficiently far from the boundary of $D^{(i)}$, and identically zero at the boundary.

The two way traveltime $T^{(i, j)}(\mathbf{x}, \mathbf{s}, \mathbf{r})$ is

$$
T^{(i, j)}(\mathbf{x}, \mathbf{s}, \mathbf{r})=T_{1}^{(i)}(\mathbf{x}, \mathbf{s})+T_{1}^{(j)}(\mathbf{x}, \mathbf{r})
$$


In two dimensions we can define the scattering angle $\theta^{(i, j)}(\mathbf{x}, \mathbf{s}, \mathbf{r})$ by

$$
\theta^{(i, j)}(\mathbf{x}, \mathbf{s}, \mathbf{r})=\alpha^{(j)}(\mathbf{x}, \mathbf{r})-\alpha^{(i)}(\mathbf{x}, \mathbf{s})
$$

For a definition of scattering angle - azimuth appropriate for three dimensions, see Burridge and Beylkin (1988). These functions are defined for $(\mathbf{x}, \mathbf{s}, \mathbf{r})$ in a domain $D^{(i, j)}$ consisting of points $(\mathbf{x}, \mathbf{s}, \mathbf{r})$ with $(\mathbf{x}, \mathbf{s}) \in D^{(i)}$ and $(\mathbf{x}, \mathbf{r}) \in D^{(j)}$.

A cutoff function for the domain $D^{(i, j)}$ is given by

$$
A^{(i, j)}(\mathbf{x}, \mathbf{s}, \mathbf{r})=A_{1}^{(i)}(\mathbf{x}, \mathbf{s}) A_{1}^{(j)}(\mathbf{x}, \mathbf{r}) .
$$

For the purposes of this paper, the Kirchhoff imaging formula is

$$
f(\mathbf{x})=\sum_{i, j} \int \mathrm{d} s \mathrm{~d} r A^{(i, j)}(\mathbf{x}, \mathbf{r}, \mathbf{s})\left(H^{n-1-\sigma^{(i)}-\sigma^{(j)}} d\right)\left(\mathbf{r}, \mathbf{s}, T^{(i, j)}(\mathbf{x}, \mathbf{r}, \mathbf{s})\right) .
$$

Here $n=2$ for $2 \mathrm{D}, n=3$ for $3 \mathrm{D}$. The output is a single image function $f(\mathbf{x})$ of position - that is, this formula implements migration plus postmigration stack. The operator $H$ appearing in the integrand is the Hilbert transform with respect to time, and $\sigma^{(i)}$ is the KMAH index of the $i$ th traveltime branch, to account for the phase shift that occurs when the rays go through a caustic (note that the term $H^{n-1-\sigma^{(i)}-\sigma^{(j)}} d$ in equation (12) can be written as $\pm d$ or $\pm H d$ by using that $H^{2}$ is minus the identity).

As mentioned above, Ten Kroode et al. (1998) explained that this formula produces a correct image, with reflectors in the right places and not elsewhere, provided that the socalled Traveltime Injectivity Condition ("TIC") is satisfied. This condition is satisfied for both examples presented in the paper, and indeed our theoretical development assumes it. See Ten Kroode et al. (1998) for examples in which TIC fails, Nolan and Symes (1997) for an extension of the TIC concept to general acquisition geometries, and Stolk (2000) for a less restrictive condition which also assures correct imaging.

Many variants of this formula have been proposed, either restricting the traveltime branches appearing in the sum or adding amplitude factors to attempt to achieve "true" 
reflection amplitude in the output image. As we have noted in the introduction, neglect of traveltime branches generally degrades the image (Geoltrain and Brac, 1993; Operto et al., 2000). Variants taking into account all traveltime branches are kinematically equivalent to equation (12), i.e. produce reflectors with the same locations and orientations.

Various Kirchhoff prestack migration formulas are obtained from equation (12) by restricting the integral over $(\mathbf{s}, \mathbf{r})$ to a constant value of the image (or bin or gather) parameter. The latter can be viewed as a function $\mathbf{e}$ of $(\mathbf{x}, \alpha, \beta)$, and hence as a (possibly) multivalued function $\mathbf{e}^{(i, j)}(\mathbf{x}, \mathbf{s}, \mathbf{r})$. Three important examples are $\mathbf{e}^{(i, j)}(\mathbf{x}, \mathbf{s}, \mathbf{r})=\mathbf{s}$ (common source), $\mathbf{e}^{(i, j)}(\mathbf{x}, \mathbf{s}, \mathbf{r})=\mathbf{r}-\mathbf{s}$ (common offset), and $\mathbf{e}^{(i, j)}(\mathbf{x}, \mathbf{s}, \mathbf{r})=\theta^{(i, j)}(\mathbf{x}, \mathbf{s}, \mathbf{r})$ (common scattering angle). Restricting the integral (12) by inserting a $\delta$-function of e we obtain

$$
\begin{aligned}
f(\mathbf{x}, \mathbf{e})=\sum_{i, j} \int \mathrm{d} s \mathrm{~d} r A^{(i, j)}(\mathbf{x}, \mathbf{s}, \mathbf{r}) & \\
& \times \delta\left(\mathbf{e}-\mathbf{e}^{(i, j)}(\mathbf{x}, \mathbf{s}, \mathbf{r})\right)\left(H^{n-1-\sigma^{(i)}-\sigma^{(j)}} d\right)\left(\mathbf{s}, \mathbf{r}, T^{(i, j)}(\mathbf{x}, \mathbf{s}, \mathbf{r})\right) .
\end{aligned}
$$

That is, the output is a function $f(\mathbf{x}, \mathbf{e})$ of position $\mathbf{x}$ and bin parameter $\mathbf{e}$. A further integration over e produces $f(\mathbf{x})$ (output of equation (12)). So $f(\mathbf{x}, \mathbf{e})$ is the unstacked image volume, expressed in a generic way that applies to all of the binning schemes mentioned above.

\section{Imaging equations}

Suppose a reflector at a subsurface point $\mathbf{x}$ leads to an event (wave front) at a position in the data given by $\left(\mathbf{s}, \mathbf{r}, t_{\mathrm{s}}+t_{\mathrm{r}}\right)$, with slowness $\left(\mathbf{p}_{\mathrm{s}}, \mathbf{p}_{\mathrm{r}}\right)$. In (Stolk, 2002) equations have been derived that describe the points $(\overline{\mathbf{x}}, \overline{\mathbf{e}})$ in the image volume (i.e. the output $f(\mathbf{x}, \mathbf{e})$ in equation (13)) at which this event leads to an apparent reflector. The apparent reflector 
coordinates $(\overline{\mathbf{x}}, \overline{\mathbf{e}})$ correspond one-to-one with the solutions $\left(\overline{\mathbf{x}}, \mathbf{p}_{\mathrm{e}}\right)$ of the equations

$$
\begin{array}{r}
T^{(i, j)}(\overline{\mathbf{x}}, \mathbf{s}, \mathbf{r})=t_{\mathrm{s}}+t_{\mathrm{r}} \\
-\left\langle\mathbf{p}_{\mathrm{e}}, \nabla_{\mathbf{s}} \mathbf{e}^{(i, j)}(\overline{\mathbf{x}}, \mathbf{s}, \mathbf{r})\right\rangle+\nabla_{\mathbf{s}} T^{(i)}(\overline{\mathbf{x}}, \mathbf{s}, \mathbf{r})=\mathbf{p}_{\mathrm{s}} \\
-\left\langle\mathbf{p}_{\mathrm{e}}, \nabla_{\mathbf{r}} \mathbf{e}^{(i, j)}(\overline{\mathbf{x}}, \mathbf{s}, \mathbf{r})\right\rangle+\nabla_{\mathbf{r}} T^{(i)}(\overline{\mathbf{x}}, \mathbf{s}, \mathbf{r})=\mathbf{p}_{\mathrm{r}}
\end{array}
$$

Here $\mathbf{p}_{\mathrm{e}}$ is equal to the $\mathbf{e}$-wave vector divided by the frequency $\omega$ (the $e$-slowness or move$o u t$ ). The bin parameter (angle or offset) of the wave front is given by $\overline{\mathbf{e}}=\mathbf{e}^{(i, j)}(\overline{\mathbf{x}}, \mathbf{s}, \mathbf{r})$. Together, the equations (14) consist of $2 n-1$ equations for $2 n-1$ unknows, with no obvious dependencies. [Again, $n$ denotes space dimension, $n=2$ for 2D imaging, $n=3$ for 3D imaging.] So one might guess that the solutions are stable, and indeed this is generally the case. As explained by Stolk (2002) one set of solutions corresponds to the correct image, with $(i, j)$ corresponding to the raypaths of the data $\left(\mathbf{s}, \mathbf{r}, \mathbf{p}_{\mathrm{s}}, \mathbf{p}_{\mathrm{r}}, t_{\mathrm{s}}+t_{\mathrm{r}}\right)$, and $\overline{\mathbf{x}}=\mathbf{x}, \mathbf{p}_{\mathrm{e}}=\mathbf{0}$ (zero e move-out). In addition there may be other sets of solutions the artifacts of the title - that must satisfy $\mathbf{p}_{\mathrm{e}} \neq \mathbf{0}$ in view of TIC (see Stolk (2002)).

If $\mathbf{e}=$ offset, the equations (14) simplify to $n$ equations for $\overline{\mathbf{x}}$ and $\mathbf{p}_{\mathrm{e}}=\overline{\mathbf{p}}_{\mathrm{s}}+\overline{\mathbf{p}}_{\mathrm{r}}$ :

$$
\begin{array}{r}
T^{(i, j)}(\overline{\mathbf{x}}, \mathbf{s}, \mathbf{r})=t_{\mathrm{s}}+t_{\mathrm{r}} \\
\overline{\mathbf{p}}_{\mathrm{s}}+\overline{\mathbf{p}}_{\mathrm{r}}=\mathbf{p}_{\mathrm{s}}+\mathbf{p}_{\mathrm{r}} .
\end{array}
$$

Appendix B describes the numerical methods used to solve the equations (14) in the offset and angle cases.

\section{Numerical implementation of migration formulas}

This section describes a numerical implementation of equation (13). The authors make no pretense that this implementation is optimal in any way, other than that it appears to approximate equation (13) with sufficient accuracy to illustrate the contentions in this paper. 
For the remainder of this Appendix and the next, we will adopt specifically 2D notation - $s$ and $r$ will be the horizontal coordinates of the source and receiver positions, $\mathbf{x}=(x, z)$, etc. The bin coordinate $e$ is also scalar, e.g. the offset $r-s$ or the scattering angle.

Numerical implementation of equation (13) requires discrete approximations to the one-way traveltime function, cutoff function, angle function $T_{1}^{(i)}(\mathbf{x}, s), A_{1}^{(i)}(\mathbf{x}, s), \alpha^{(i)}(\mathbf{x}, s)$ for all values of the branch index $i$, and the integration in equation (13). The two-way quantities can then be calculated from equations (9), (10), (11).

Suppose data is given on a regular grid of $(s, r, t)$ values with grid intervals (or interval vectors, for 3D problems) $\Delta s, \Delta r$ and $\Delta t$, possibly after binning from irregularly sampled $s$ and $r$. Image location and bin parameter occupy a regular grid of $(\mathbf{x}, \mathbf{e})$ values. Gridded values of these coordinates will be denoted by $\hat{s}, \hat{r}, \hat{t}, \hat{\mathbf{x}}, \hat{\mathbf{e}}$ respectively.

In our implementation of equation (13), we used numerical ray tracing to compute approximations to $T_{1}^{(i)}(\hat{\mathbf{x}}, \hat{s}), A_{1}^{(i)}(\hat{\mathbf{x}}, \hat{s}), \alpha^{(i)}(\hat{\mathbf{x}}, \hat{s})$ and the KMAH indices $\sigma^{(i)}$. For each pair $(\hat{\mathbf{x}}, \hat{s})$ we kept all arrivals for which the definition domain $D^{(i)}$ was reasonably large (up to three in the lens, more for the Marmousi example). This tactic was adequate for the examples presented below; the task of writing a general purpose code along these lines must be formidable.

Integrations over $s, r$ are approximated by the trapezoidal rule. For time $t$ not in the grid, the (possibly) Hilbert transformed data is approximated by linear interpolation. The $\delta$-function in $e$ is approximated by adjoint interpolation. In the $2 \mathrm{D}$ imaging case, $e$ is a scalar variable, sampled at interval $\Delta e$. Let $e_{\text {int }}$ denote integer part of $e^{(i, j)}(\hat{\mathbf{x}}, \hat{s}, \hat{r}) / \Delta e$, and $e_{\mathrm{frac}}=e^{(i, j)}(\hat{\mathbf{x}}, \hat{s}, \hat{r}) / \Delta e-e_{\mathrm{int}}$. In this notation a discrete approximation $\hat{\delta}$ to $\delta(e-$ $\left.e^{(i, j)}(\hat{\mathbf{x}}, \hat{s}, \hat{r})\right)$ is given by

$$
\hat{\delta}(\hat{e}, \hat{\mathbf{x}}, \hat{s}, \hat{r}):=\frac{\left(1-e_{\mathrm{frac}}\right) \delta_{K}\left(\hat{e}, e_{\mathrm{int}}\right)+e_{\mathrm{frac}} \delta_{K}\left(\hat{e}, e_{\mathrm{int}}+1\right)}{\Delta e}
$$

where $\delta_{K}$ is the Kronecker $\delta$ on the $\Delta e$ lattice. A similar approximation is possible for 
the $3 D$ case.

With these approximations, the discrete version of (13) is

$$
\hat{f}(\hat{\mathbf{x}}, \hat{e})=\Delta s \Delta r \sum_{i, j} \sum_{\hat{s}, \hat{r}} A^{(i, j)}(\hat{\mathbf{x}}, \hat{s}, \hat{r}) \hat{\delta}(\hat{e}, \hat{\mathbf{x}}, \hat{s}, \hat{r})\left(H^{n-1-\sigma^{(i)}-\sigma^{(j)}} d\right)\left(\hat{s}, \hat{r}, T^{(i, j)}(\hat{\mathbf{x}}, \hat{s}, \hat{r})\right)
$$

The discrete version of expression (12) for the stacked image is

$$
\hat{f}_{\text {stack }}(\hat{\mathbf{x}})=\Delta s \Delta r \sum_{i, j} \sum_{\hat{s}, \hat{r}} A^{(i, j)}(\hat{\mathbf{x}}, \hat{s}, \hat{r})\left(H^{n-1-\sigma^{(i)}-\sigma^{(j)}} d\right)\left(\hat{s}, \hat{r}, T^{(i, j)}(\hat{\mathbf{x}}, \hat{s}, \hat{r})\right) .
$$

It follows from $(16)$ that $\hat{f}_{\text {stack }}(\hat{\mathbf{x}})=\Delta e \sum_{\hat{e}} \hat{f}(\hat{\mathbf{x}}, \hat{e})$.

Implementation consisted of two stages. The first stage employed numerical ODE solvers and interpolation routines in a general purpose mathematical software package (Mathematica $\left.^{T M}\right)$ to compute the gridded traveltime data $\left(T_{1}^{(i)}(\hat{\mathbf{x}}, \hat{s}), A_{1}^{(i)}(\hat{\mathbf{x}}, \hat{s}), \alpha^{(i)}(\hat{\mathbf{x}}, \hat{s})\right.$, $\left.\sigma^{(i)}\right)$ and stored the results to disk files. The second stage consisted of a program, written in $\mathrm{C}$, which read the traveltime data and the input seismograms from disk, and evaluated the summation in equation (17), storing its results to disk as well.

For the lens model evaluations of equation (17), the one-way traveltimes $T_{1}^{(i)}(\mathbf{x}, s)$, a cutoff $A^{(i)}(\mathbf{x}, s)$ and the one-way angle function $\alpha^{(i)}(\mathbf{x}, s), i=1,2,3$, were computed on the domain $(x, z, s) \in[0,1] \times[1.5,2.5] \times[-2,2] \mathrm{km}^{3}$, with grid interval $0.01 \mathrm{~km}$ in all three directions. For Marmousi the computation was done for constant $x=6.2 \mathrm{~km}$ with $(z, s)$ in the domain $[3.2,9.0] \times[2.1,2.7] \mathrm{km}^{2}$, with grid interval $0.02 \mathrm{~km}$ in the $z$ direction and $0.025 \mathrm{~km}$ in the $s$ direction.

\section{APPENDIX B}

\section{NUMERICAL SOLUTION OF THE IMAGING EQUATIONS}

This appendix gives a detailed account of the methods used to solve the imaging equations (14), for both the common offset and common angle cases. 


\section{Common Offset}

The first equation in the equations (15) implies that there are rays connecting $s$ and $r$ to the imaging point $\overline{\mathbf{x}}$ with total traveltime that equals $t_{\mathrm{s}}+t_{\mathrm{r}}$. Denote by $\bar{p}_{\mathrm{s}}$ and $\bar{p}_{\mathrm{r}}$ the horizontal slownesses of the rays at the surface. It follows that there must be $t$ such that

$$
F\left(s, r, \bar{p}_{\mathrm{s}}, \bar{p}_{\mathrm{r}}, t\right):=\mathbf{x}\left(s, \bar{p}_{\mathrm{s}}, t_{\mathrm{s}}-t\right)-\mathbf{x}\left(r, \bar{p}_{\mathrm{r}}, t_{\mathrm{r}}+t\right)=0
$$

Here $\mathbf{x}(s, p, t)$ is the position at time $t$ along the ray with initial conditions at the surface determined by $s, p$.

The second equation in equations (15) implies that there is $p$ such that $\bar{p}_{\mathrm{s}}=p_{\mathrm{s}}-p, \bar{p}_{\mathrm{r}}=$ $p_{\mathrm{r}}+p$. The image point $\overline{\mathbf{x}}$ is the intersection point of the rays with surface initial conditions determined by $\left(s, p_{\mathrm{s}}-p\right),\left(r, p_{\mathrm{r}}+p\right)$. Therefore artifacts correspond to solutions $t, p, p \neq 0$, to the following equations for the coordinates $\overline{\mathbf{x}}_{1}$ and $\overline{\mathbf{x}}_{2}$ of the intersection point:

$$
\mathbf{x}\left(s, p_{\mathrm{s}}-p, t_{\mathrm{s}}-t\right)=\mathbf{x}\left(r, p_{\mathrm{r}}+p, t_{\mathrm{r}}+t\right) .
$$

We solve these equations using the Mathematica ${ }^{T M}$ root finder. One can make use of the fact that rays carrying significant energy in this example are increasing in depth, i.e. that $t$ is a monotone increasing function of depth along a ray, to first solve for $t$ as a function of $p$. Then a single equation for $p$ remains.

Creating a kinematic common image gather amounts to finding all solutions to equations (15) for some fixed value of the horizontal image coordinate $x$. Since the reflector is flat and specular, true reflection locations are parameterized by horizontal position $x$ and scattering angle $\theta$. Each solution of equations (15) - i.e. each apparent reflector, true or artifact, in the migrated image volume - corresponds to a true reflecting position which generated the data event which it images. So we can parameterize the solutions of equations $(15)$ by the true reflection position $(x, \theta)$ to which it corresponds. In these 
calculations we use the multivalued traveltime database subsequently used for prestack migration.

For each pair of potential true reflection parameters $(x, \theta)$, we compute the surface event coordinates $s, r, p_{\mathrm{s}}, p_{\mathrm{r}}, t_{\mathrm{s}}+t_{\mathrm{r}}$ as functions of $x$ and $\theta$. Since the horizontal coordinate of the image $\bar{x}_{1}$ is prescribed, and the time is increasing with depth along both incident and reflected rays in the region of interest, the traveltime equation in equations (15) uniquely fixes the depth coordinate $\bar{z}(x, \theta)$. The $p_{\mathrm{s}}, p_{\mathrm{r}}$ equation in equations (15) now determines a subset of all $(x, \theta)$ that contributes to the CIG, i.e. we have to find all $(x, \theta)$ that satisfy

$$
0=R_{\text {offset }}^{(i, j)}(x, \theta):=\left(\frac{\partial T_{1}^{(i)}}{\partial s}(\overline{\mathbf{x}}(x, \theta), s)+\frac{\partial T_{1}^{(j)}}{\partial r}(\overline{\mathbf{x}}(x, \theta), r)\right)-\left(p_{\mathrm{s}}(x, \theta)+p_{\mathrm{r}}(x, \theta)\right)
$$

When at a zero $(x, \theta)$ of $R_{\text {offset }}^{(i, j)}(x, \theta)$ the gradient $\frac{\partial R_{\text {offset }}^{(i, j)}}{\partial(x, \theta)} \neq 0$, then the zero set of $R_{\text {offset }}^{(i, j)}$ is locally a curve. In that case the zero set can be determined in a stable manner by a numerical root-finding algorithm. Our computations were based on this assumption, which was sufficient to compute the examples given below. Note that it need not be satisfied in general (see the remark at the end of the next subsection). For the value $\bar{x}=0.3$ we computed $R_{\text {offset }}^{(i, j)}$ for a sufficiently large array of $(x, \theta)$ values. We observed that in this case the gradient was nonzero at the zeroes of $R_{\text {offset }}^{(i, j)}$, with a single exceptional point. The values of $R_{\text {offset }}^{(i, j)}$ were displayed, allowing for the approximate determination of the zero set. The zero set was then determined precisely using a numerical rootfinding algorithm. At the exceptional point, where $R_{\text {offset }}^{(i, j)}$ and its gradient were zero, an intersection of two of the zero curves occurred. In this case we determined the curves by bisection, starting from under- and overestimates of the zero. 


\section{Common Angle}

The analysis for common scattering angle binning follows the same pattern as for common offset binning. For each point on the reflector given by $x$, and opening angle $\theta$ the quantities $s, t_{\mathrm{s}}, p_{\mathrm{s}}, r, t_{\mathrm{r}}, p_{\mathrm{r}}$ can be determined as described above. First we compute rays leading to correct image and artifacts for given values of $s, t_{\mathrm{s}}, p_{\mathrm{s}}, r, t_{\mathrm{r}}, p_{\mathrm{r}}$. As explained in the previous section the traveltime equation in equations (14) implies that there must be a $t$ such that equation (19) is satisfied. Given $p_{\mathrm{s}}, \bar{p}_{\mathrm{s}}$ (the "modeling" and "imaging" values of the source slowness), there is a unique value $p_{\mathrm{e}}=p_{\mathrm{e}, \mathrm{s}}^{(i, j)}\left(s, p_{\mathrm{s}}, \bar{p}_{\mathrm{s}}, \bar{t}_{\mathrm{s}}\right)$ such that the second equation of (14) is satisfied. Similarly $p_{\mathrm{e}}=p_{\mathrm{e}, \mathrm{r}}^{(i, j)}\left(r, p_{\mathrm{r}}, \bar{p}_{\mathrm{r}}, \bar{t}_{\mathrm{r}}\right)$ satisfying the third equation can be found. The two must be the same, which leads to the equation

$$
0=p_{\mathrm{e}, \mathrm{s}}^{(i, j)}\left(s, p_{\mathrm{s}}, \bar{p}_{\mathrm{s}}, t_{\mathrm{s}}-t\right)-p_{\mathrm{e}, \mathrm{r}}^{(i, j)}\left(r, p_{\mathrm{r}}, \bar{p}_{\mathrm{r}}, t_{\mathrm{r}}+t\right)
$$

Equations (19) and (22) form 3 equations for the 3 variables $\bar{p}_{\mathrm{s}}, \bar{p}_{\mathrm{r}}, t$ (in 2D). The position

$\overline{\mathbf{x}}$ is given by $\mathbf{x}\left(s, \bar{p}_{\mathrm{s}}, t_{\mathrm{s}}-t\right)=\mathbf{x}\left(r, \bar{p}_{\mathrm{r}}, t_{\mathrm{r}}+t\right)$, the angle is computed from the directions of the imaging rays at the intersection point.

The numerical rootfinding for these equations was done in two steps. If $\xi$ is a direction (vector) with positive inner product with the velocity vectors of the rays to both source and receiver, then the inner product of $\xi \cdot F(\ldots, t)$ is decreasing with $t$, and the solution to $\xi \cdot F=0$ is a straightforward rootfinding exercise. Then two equations remain with unknowns $p_{\mathrm{s}}, p_{\mathrm{r}}$. By computing the values for an array of $p_{\mathrm{s}}, p_{\mathrm{r}}$ the solutions can be found approximately. By numerical rootfinding precise solutions can then be found.

The kinematic computation of position of wave fronts in the angle CIG proceeds similarly as in the offset case. In this case, according to equation (22), the function $R_{\text {offset }}^{(i, j)}(x, \theta)$ is replaced by

$$
R_{\text {angle }}^{(i, j)}(x, \theta):=p_{\mathrm{e}, \mathrm{s}}^{(i, j)}\left(s, p_{\mathrm{s}}, \bar{p}_{\mathrm{s}}, \bar{t}_{\mathrm{s}}\right)-p_{\mathrm{e}, \mathrm{r}}^{(i, j)}\left(r, p_{\mathrm{r}}, \bar{p}_{\mathrm{r}}, \bar{t}_{\mathrm{r}}\right),
$$


where $\bar{t}_{\mathrm{s}}=T_{1}^{(i)}(\overline{\mathbf{x}}, s), \bar{p}_{\mathrm{s}}=\frac{\partial T_{1}^{(i)}}{\partial s}(\overline{\mathbf{x}}, s)$ and similar for $\bar{p}_{\mathrm{r}}, \bar{t}_{\mathrm{r}}$.

Figure 11 shows the results of this calculation for $\bar{x}=0.3$, as discussed in the text. For $\bar{x}=0$ we also tried to determine the zero set of $R_{\text {angle }}^{(i, j)}(x, \theta)$. However, we observed that the gradient of $R_{\text {angle }}^{(i, j)}(x, \theta)$ was close to zero and the determination of the zero set using our methods became difficult. 
FIG. 1. Reflected ray pair, notation.

FIG. 2. Lens model velocity distribution with a horizontal reflector.

FiG. 3. Examples of rays and wave fronts for lens model.

Fig. 4. A shot record at $s=-0.5 \mathrm{~km}$ for lens model.

FIG. 5. Common offset (or angle) migration stack image for lens model.

FIG. 6. Some rays corresponding to image and artifact for a reflecting element at $(x, z)=(0.303,2) \mathrm{km}$, together with image of area of interest.

FIG. 7. Image from offset $1 \mathrm{~km}$ superimposed with the rays from Figure 6.

FiG. 8. An offset CIG at $x=0.3 \mathrm{~km}$, kinematic prediction and synthetic data image.

FIG. 9. Isochron, and rays leading to artifacts and correct image for reflections at $(0.3,2)$ $\mathrm{km}$, opening angle 40 degrees (a) and for reflection at $(0,2) \mathrm{km}$, opening angle 10 degrees (b).

Fig. 10. A constant angle image for angle equal to 30 degrees.

FIG. 11. An angle CIG at $x=0.3 \mathrm{~km}$, synthetic data superimposed with predicted events. 
FiG. 12. Angle CIGs, synthetic data at (a) $x=0 \mathrm{~km}$ and (b) $x=0.6 \mathrm{~km}$.

FIG. 13. Smoothed Marmousi model with 2 flat reflectors.

Fig. 14. A shot record for Marmousi model at $s=7.5 \mathrm{~km}$.

FIG. 15. Rays and wave fronts in the smoothed Marmousi model originating at

$$
(x, z)=(6.2,2.4) \mathrm{km} \text {. }
$$

FIG. 16. Branches of travel time in the take-off angle $/$ depth plane for $x=6.2 \mathrm{~km}$. A source is placed at each depth on the vertical line $x=6.2 \mathrm{~km}$, and rays are traced upwards to the surface. The branches of (one-way) traveltime at the surface are identified as a function of takeoff angle at the source, at each depth. The numbering scheme is essentially random. Take-off angle $0^{\circ}$ is horizontal in positive $x$ direction, $-90^{\circ}$ is vertically upward.

FIG. 17. An angle CIG at $x=6.2 \mathrm{~km}$. Inside the box an image artifact that is further studied. Amplitudes are clipped when they are higher than half the maximal value present.

Fig. 18. Angle CIG at $x=6.2 \mathrm{~km}$, with the pair of branches $(10,2)$ omitted from the sum. The image artifact previously present in the box is now absent.

FIG. 19. Position of artifact computed using travel time functions (line in the upper middle of the figure) together with the image (graylevel).

FIG. 20. Rays corresponding to certain events in data (solid). Rays that image these events to an artifact (dashed). The graylevel plot represents the velocity model. 


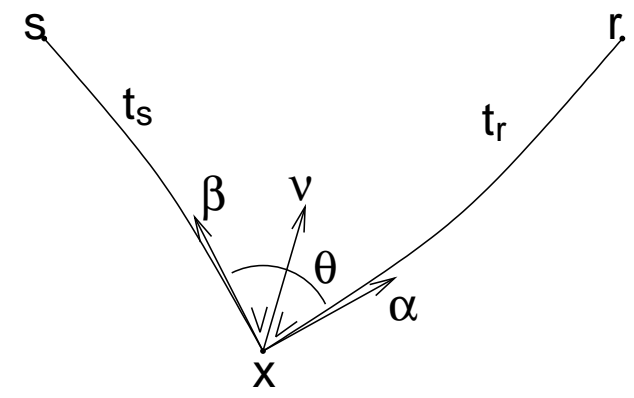

Authors: Stolk and Symes

Figure number : 1

File name(s) : raypair_schematic2.eps

Column width 


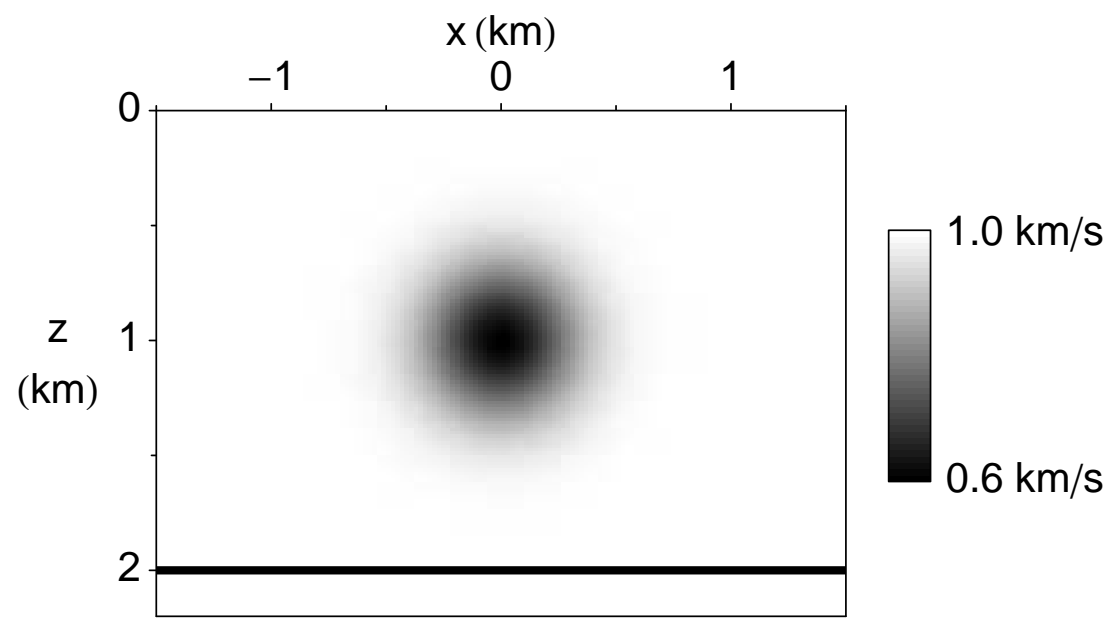

Authors: Stolk and Symes

Figure number : 2

File name(s): lensmedium.eps

Column width 


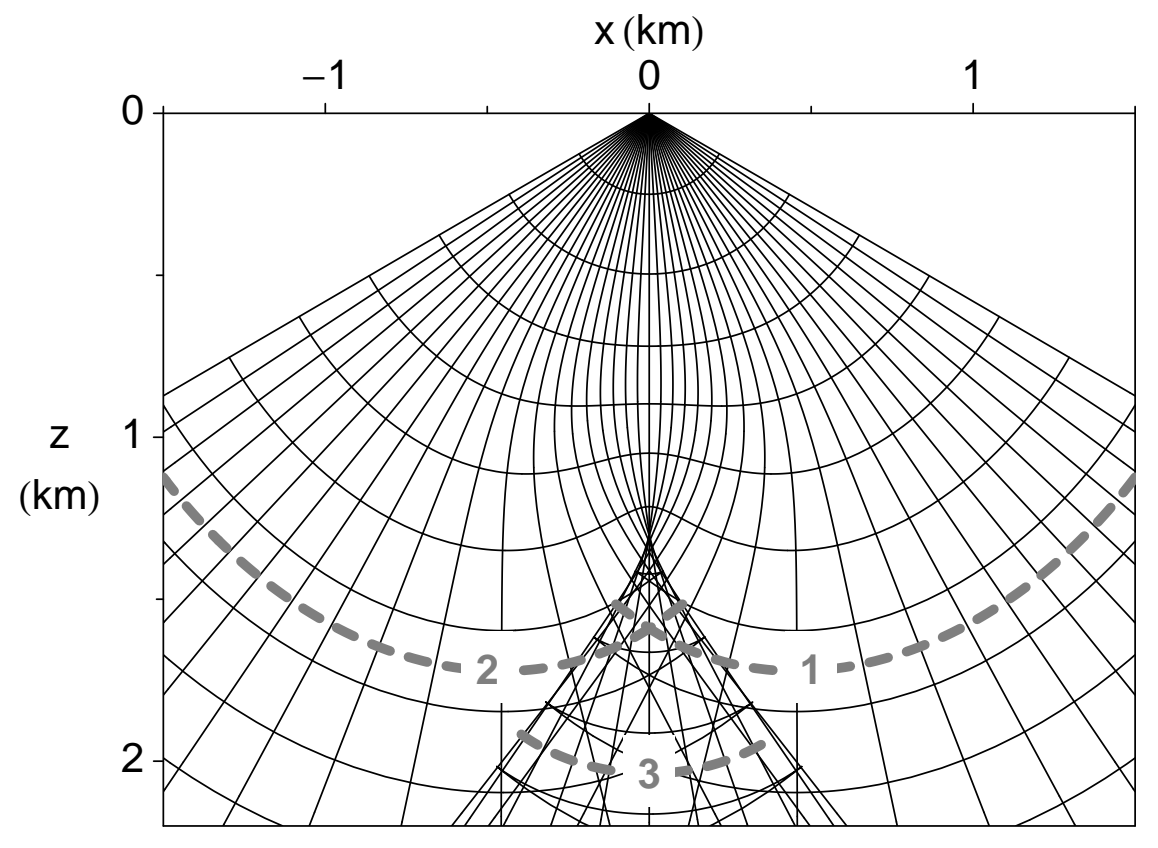

Authors: Stolk and Symes

Figure number : 3

File name(s): lensraysfrontsnocolor.eps

Column width 


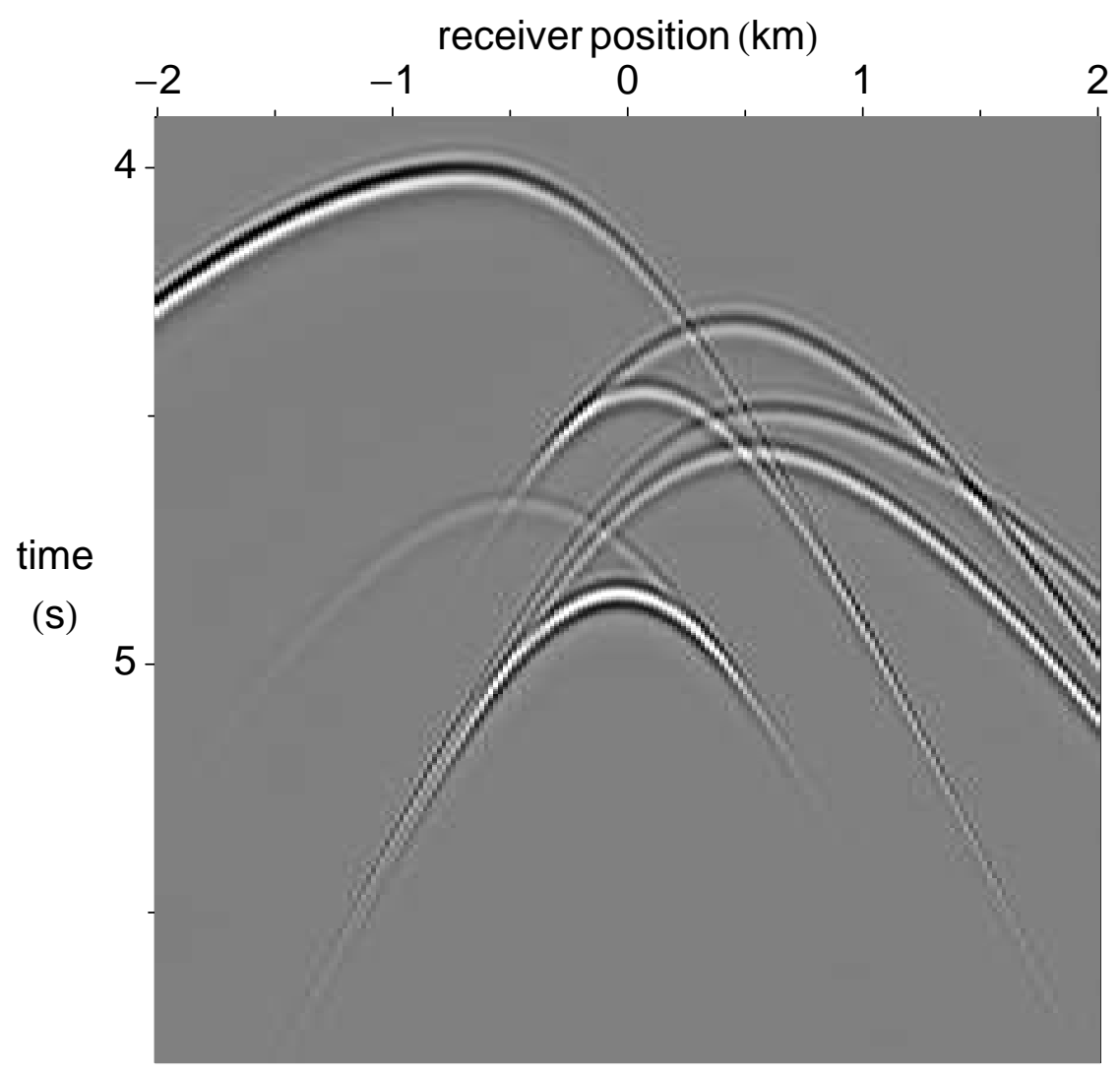

Authors: Stolk and Symes

Figure number : 4

File name(s): lensshot.eps

Column width 


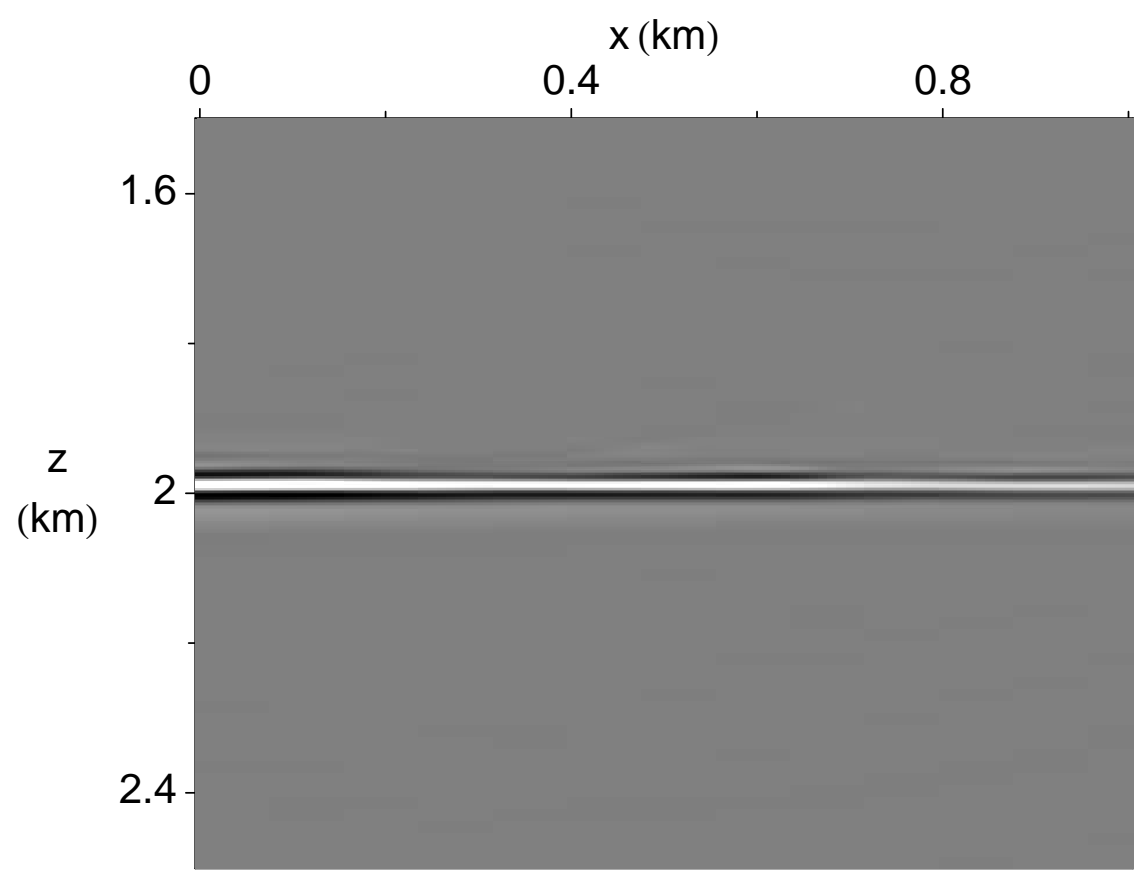

Authors: Stolk and Symes

Figure number : 5

File name(s): lensimageall.eps

Column width 


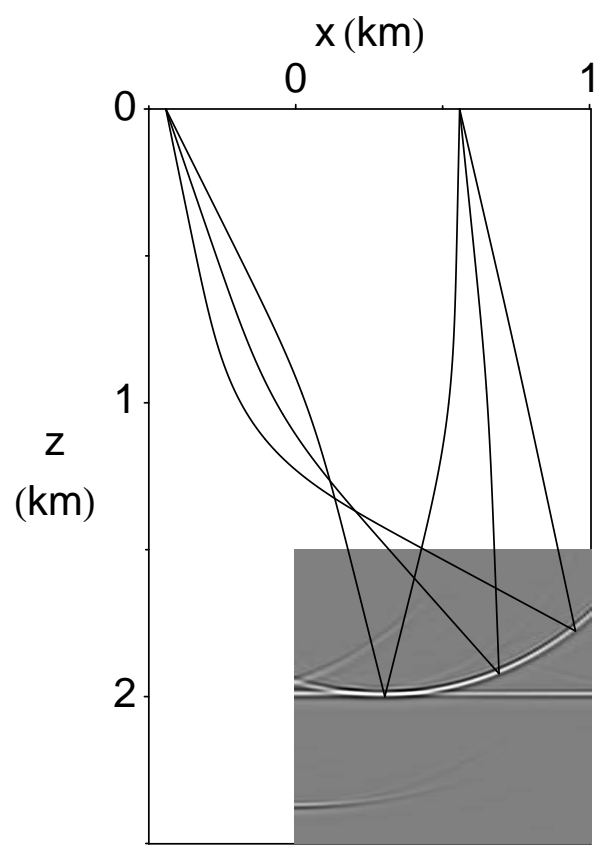

Authors: Stolk and Symes

Figure number : 6

File name(s): lensoffsetrayswithimage.eps

Column width 


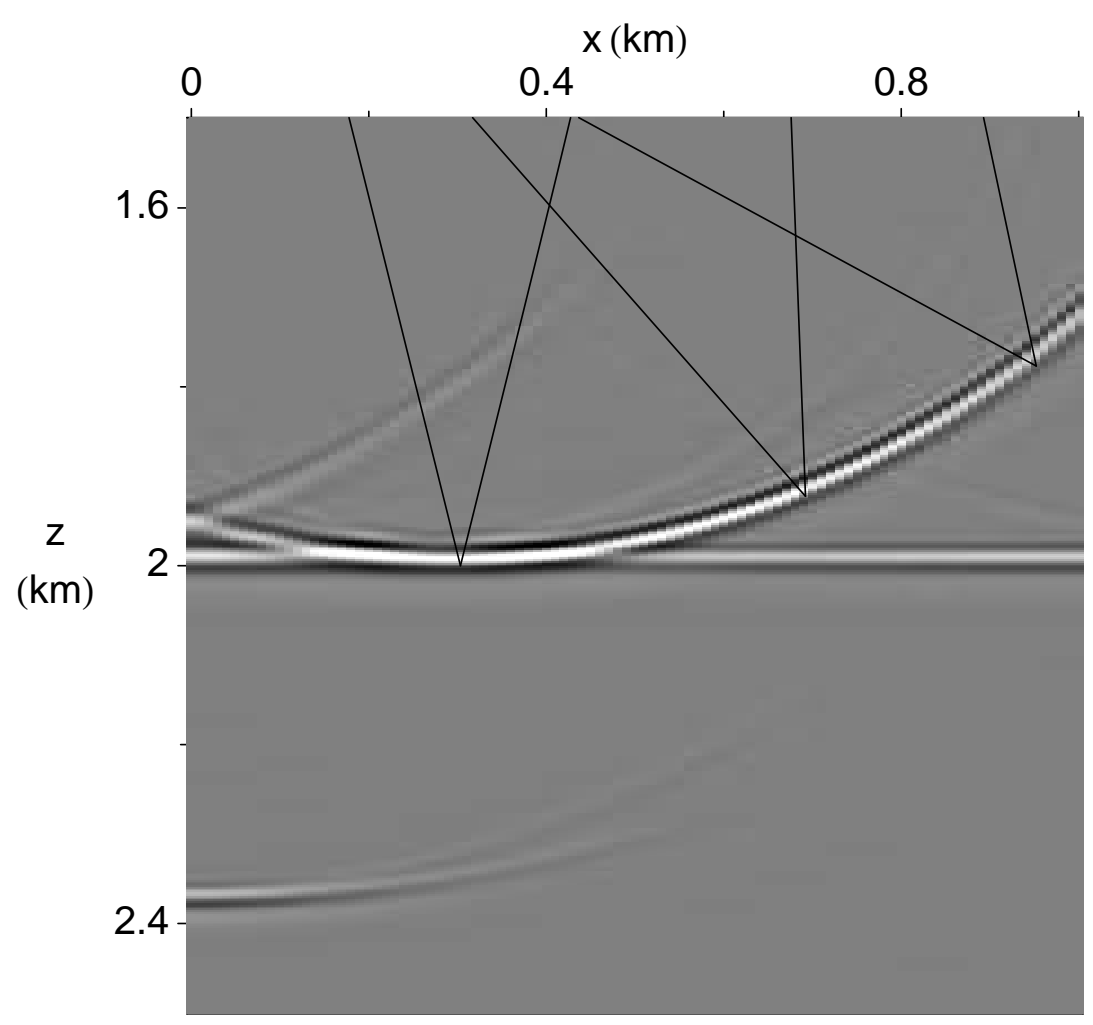

Authors: Stolk and Symes

Figure number : 7

File name(s): lensoffsetimage.eps

Column width 


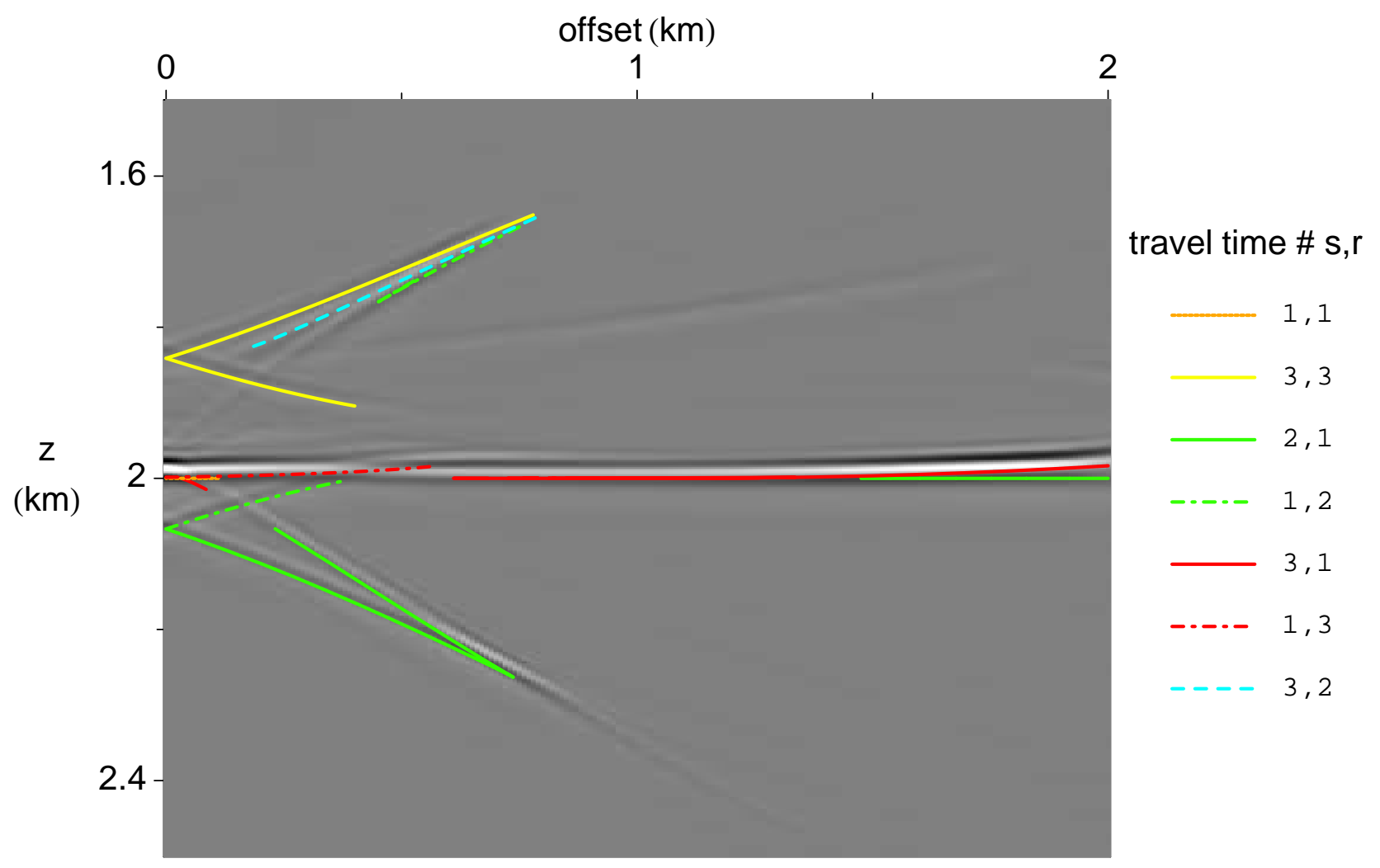

Authors: Stolk and Symes

Figure number : 8

File name(s): lensoffsetcig.eps

Page width

Color figure 

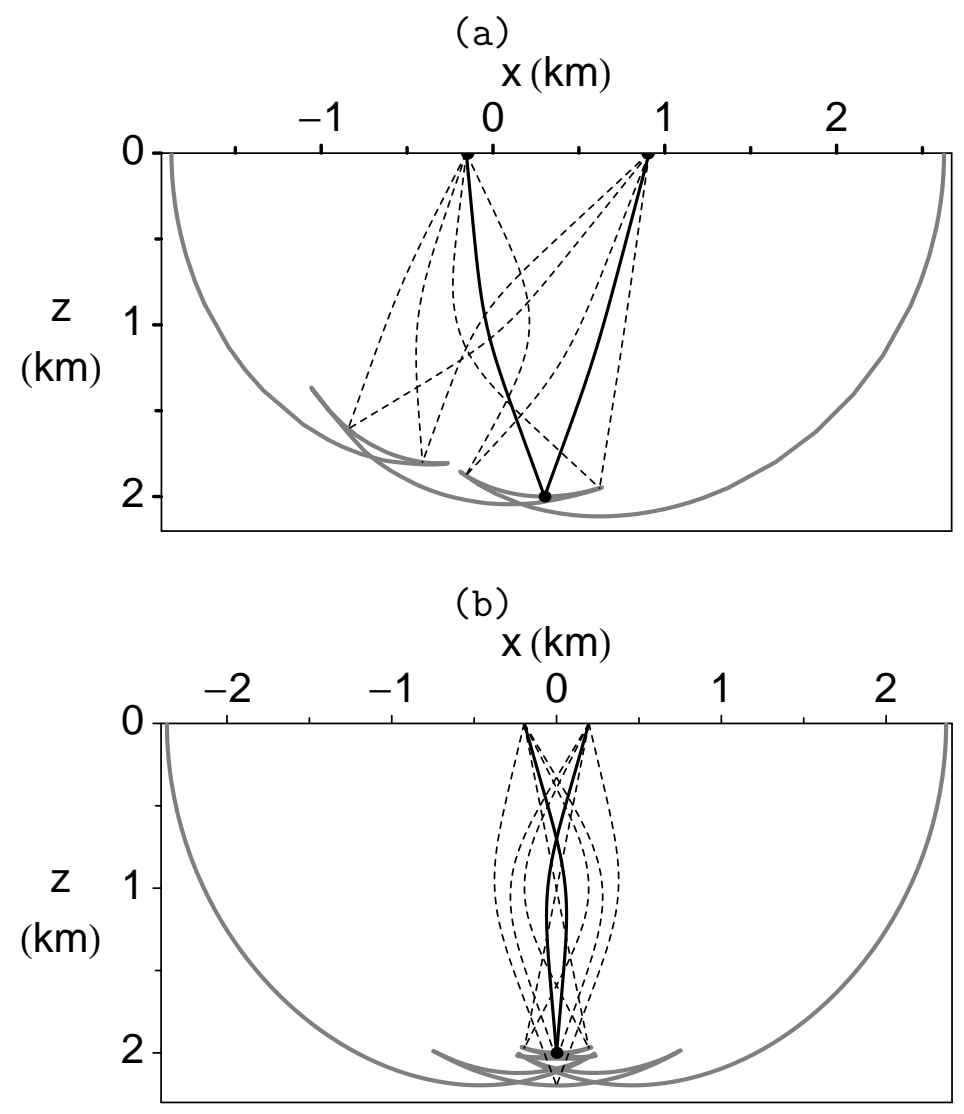

Authors: Stolk and Symes

Figure number : 9

File name(s): lensanglerays1.eps (a), lensanglerays2.eps (b) Column width 


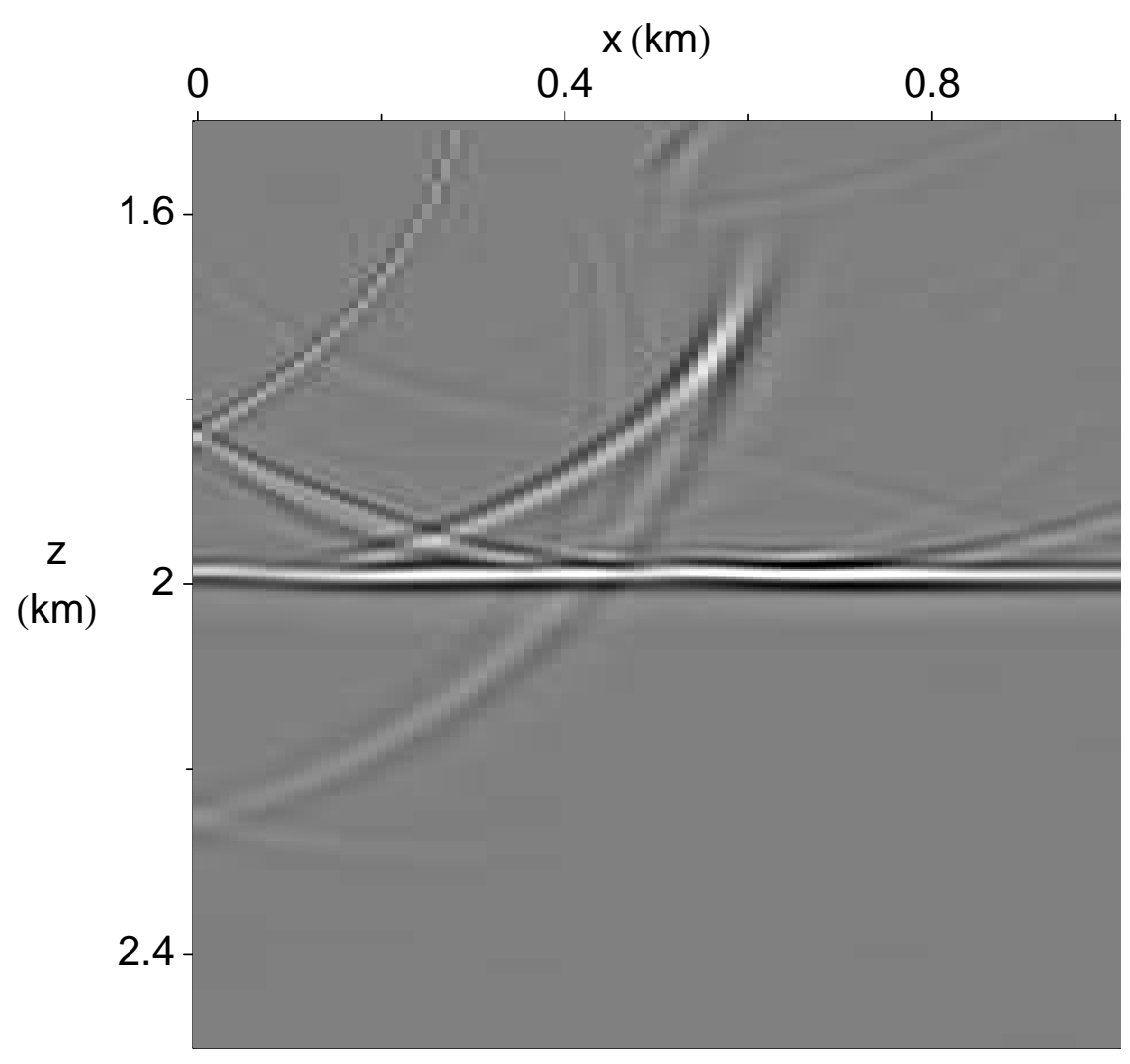

Authors: Stolk and Symes

Figure number : 10

File name(s) : lensangleimage.eps

Column width 


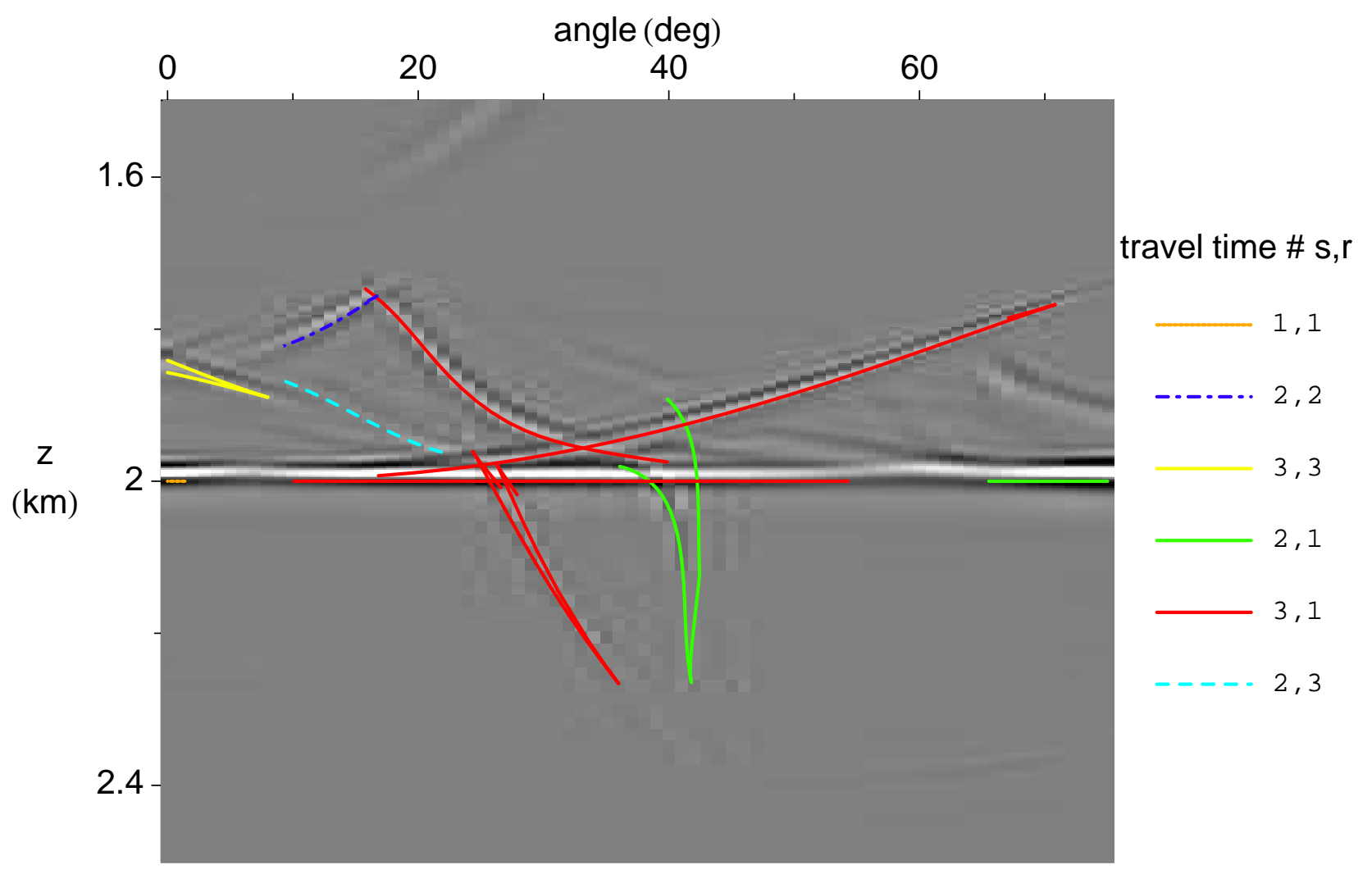

Authors: Stolk and Symes

Figure number : $\quad 11$

File name(s) : lensanglecig.eps

Page width

Color figure 

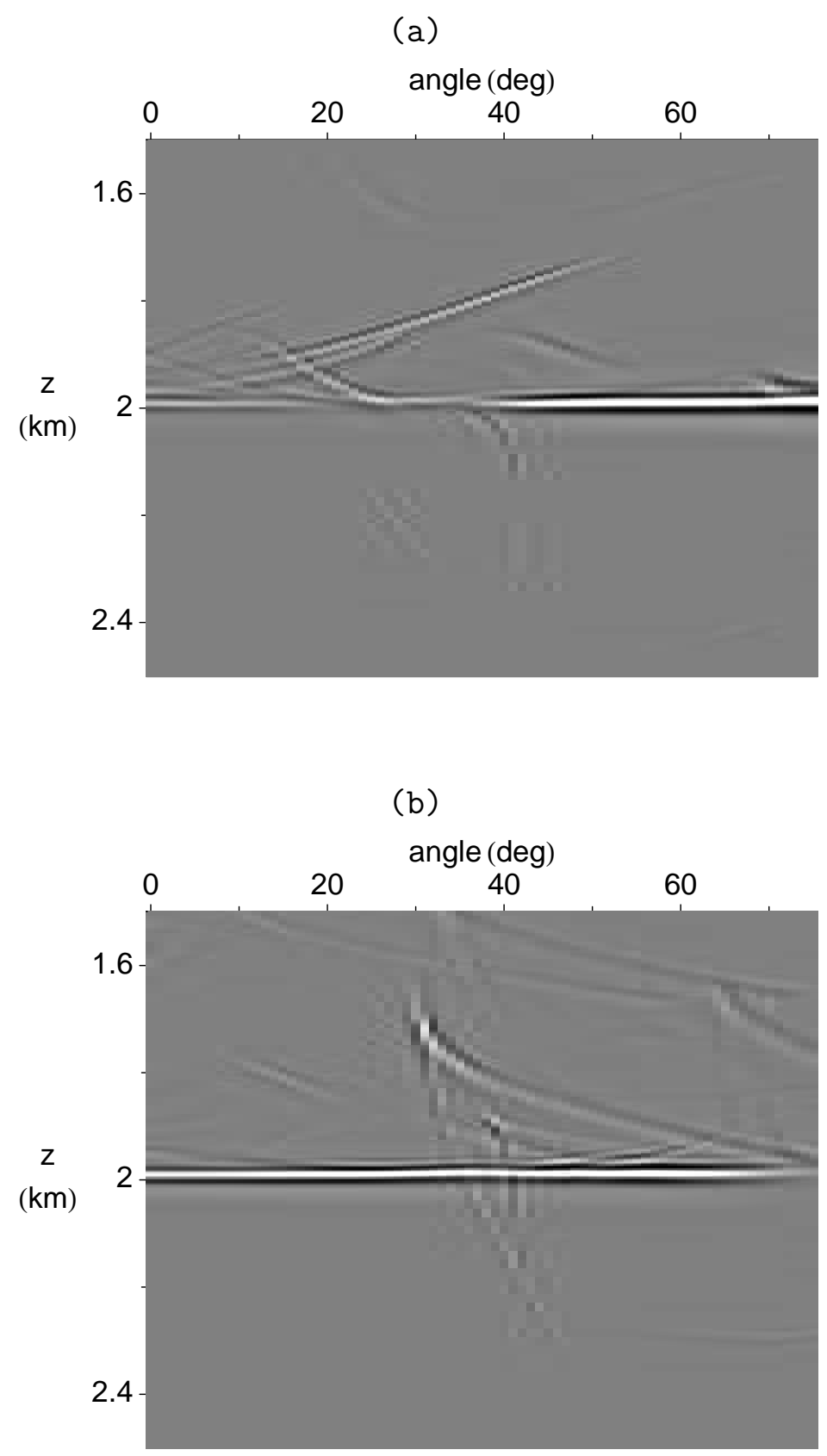

Authors: Stolk and Symes

Figure number : 12

File name(s) : lensanglecig_0_3.eps (a), lensanglecig_2_3.eps (b) Column width 


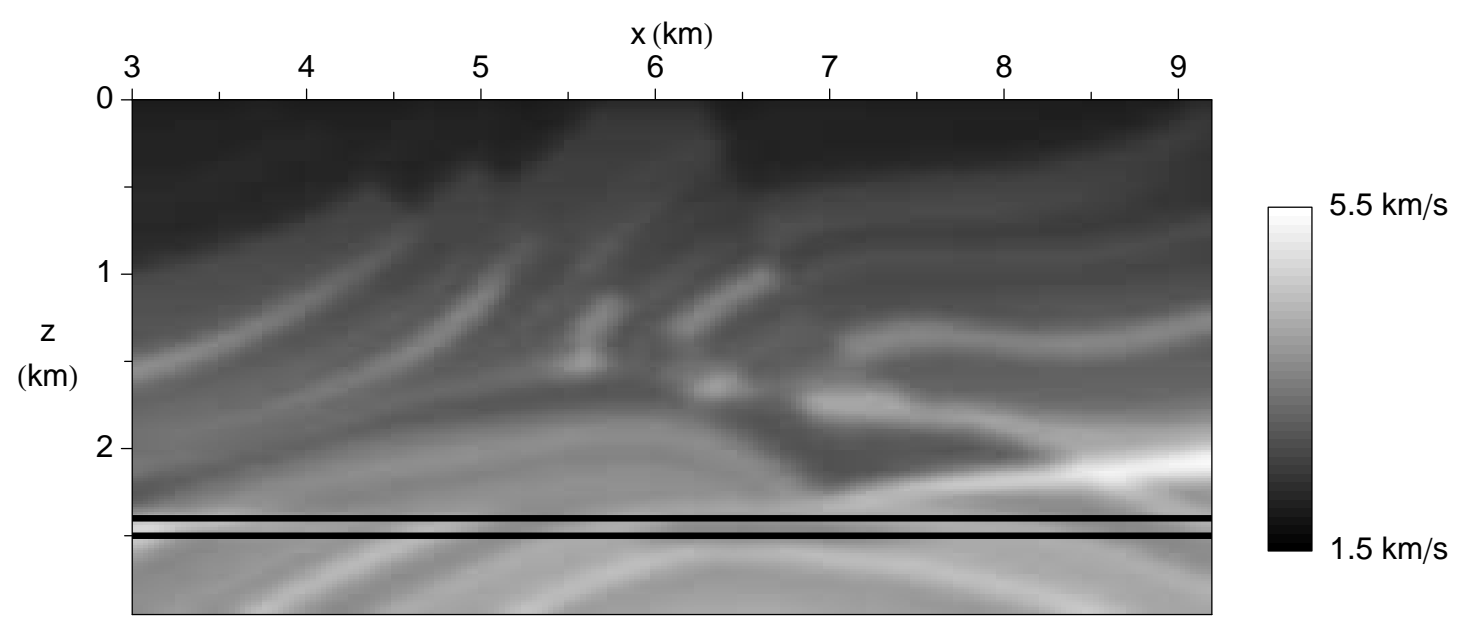

Authors: Stolk and Symes

Figure number : 13

File name(s): marmmedium.eps

Page width 


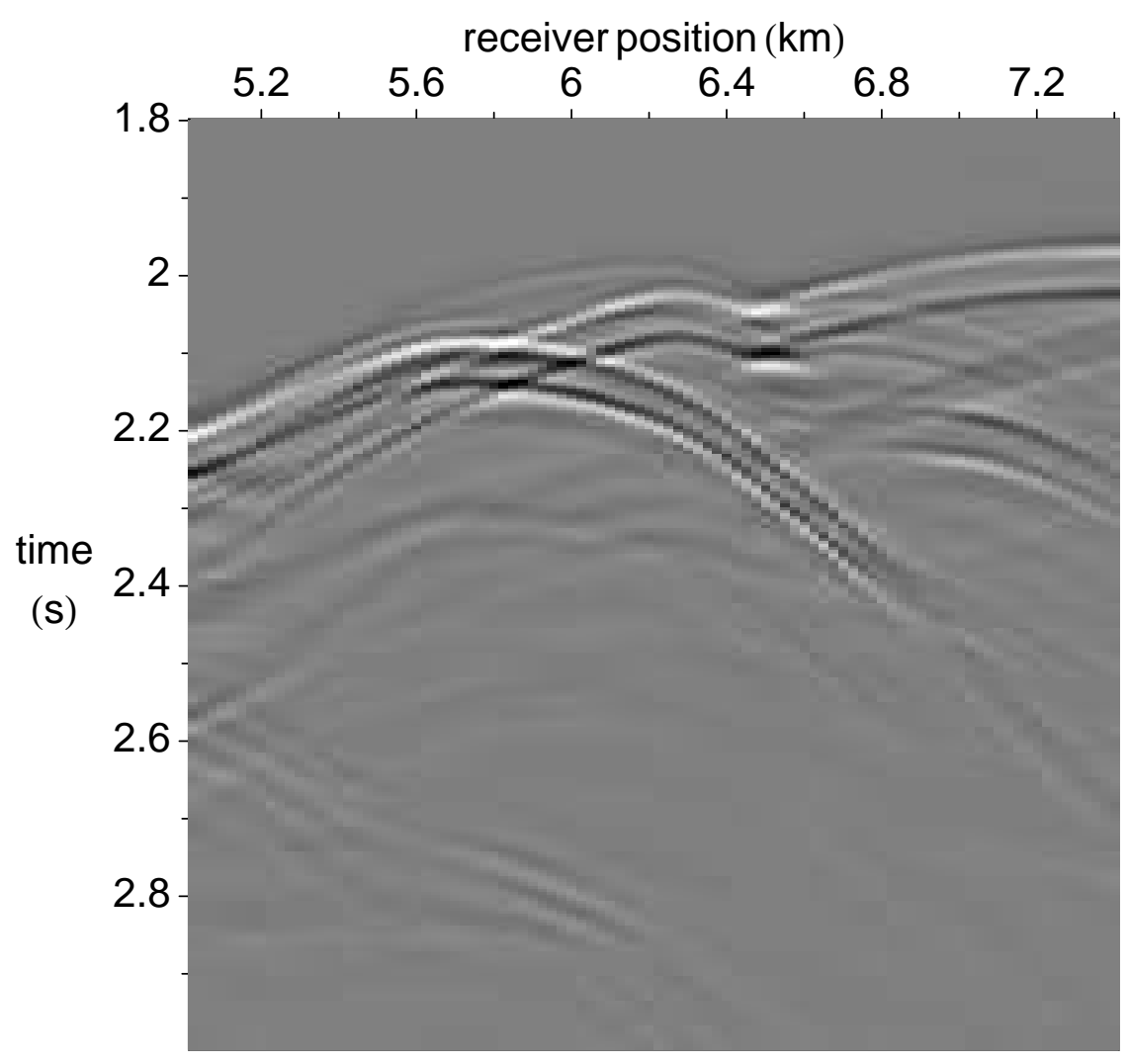

Authors: Stolk and Symes

Figure number : 14

File name(s): marmshot.eps

Column width 


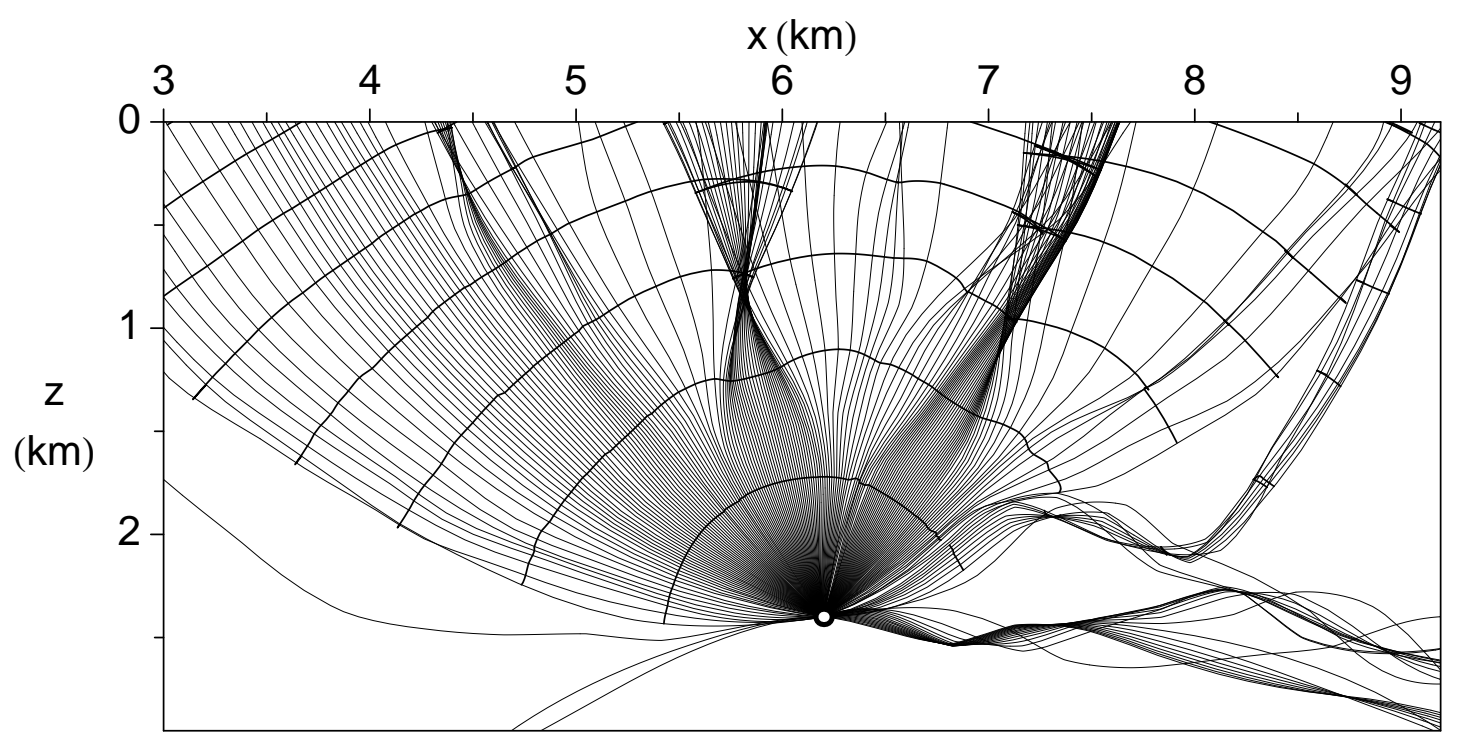

Authors: Stolk and Symes

Figure number : 15

File name(s) : marmrayswavefronts.eps

Page width 


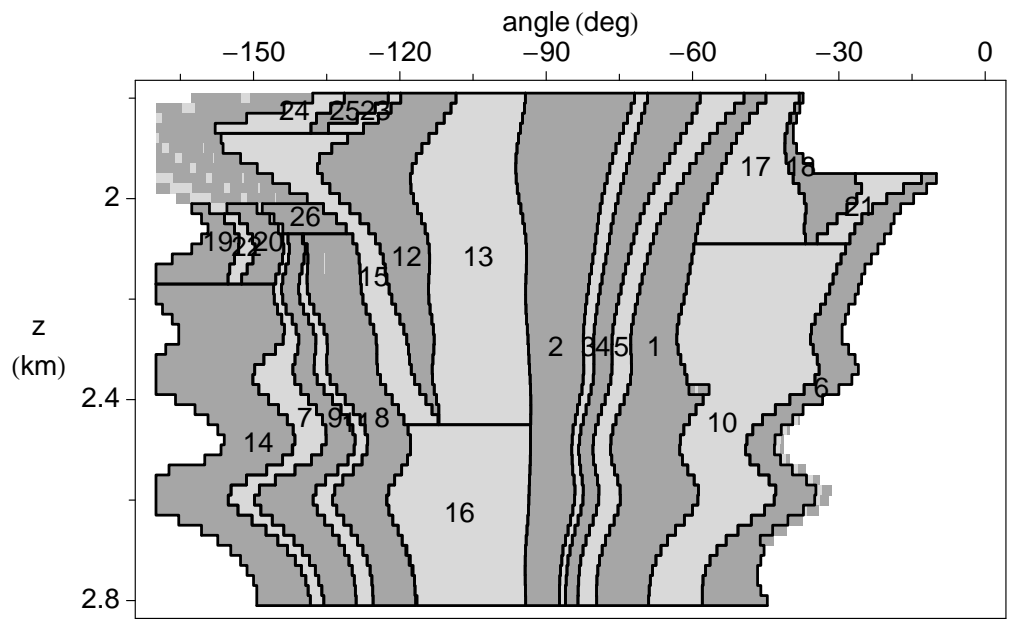

Authors: Stolk and Symes

Figure number : 16

File name(s): marmbranches.eps

Column width 


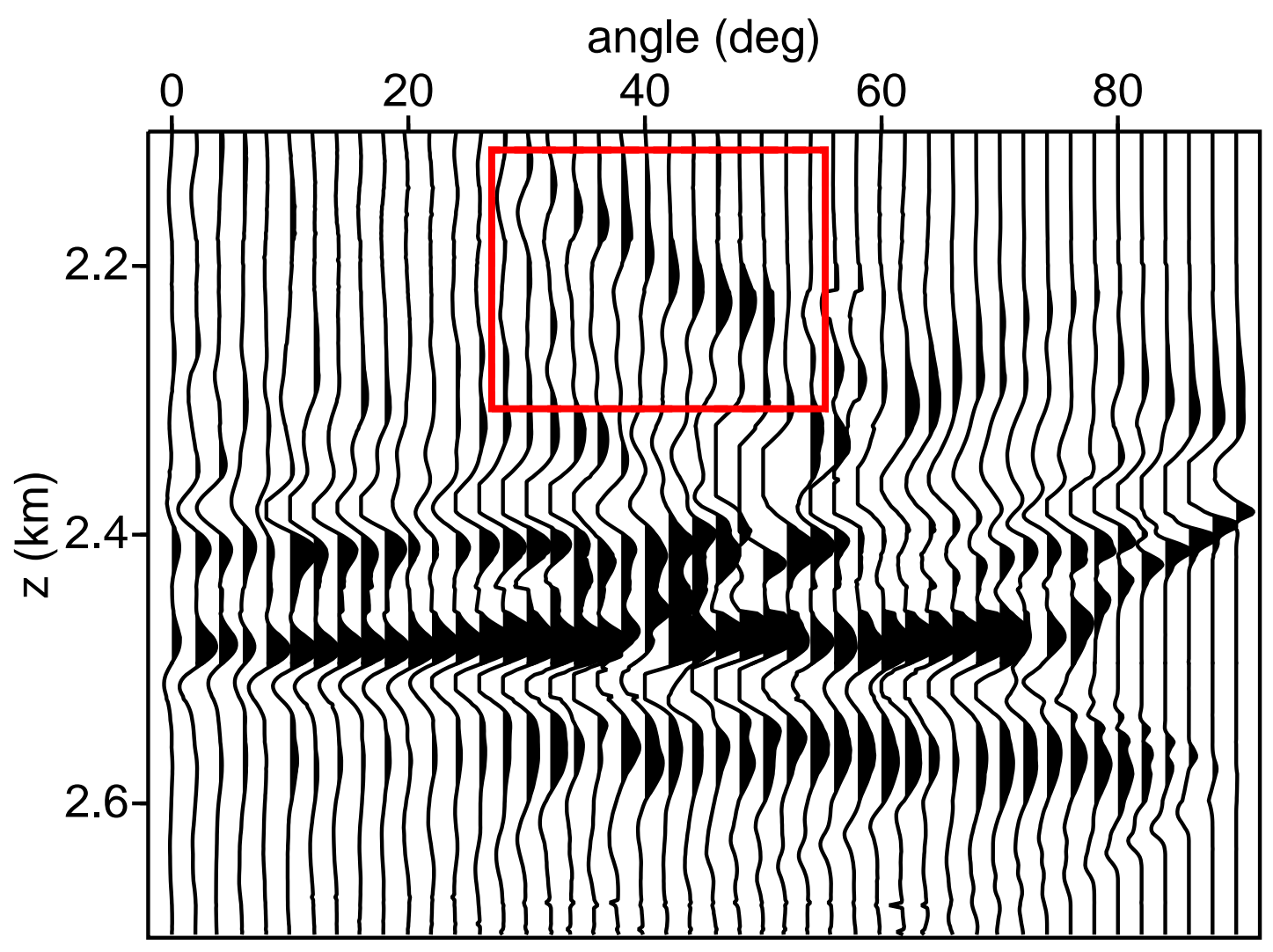

Authors: Stolk and Symes

Figure number : 17

File name(s) : marmim5box.eps

Column width

Color figure 


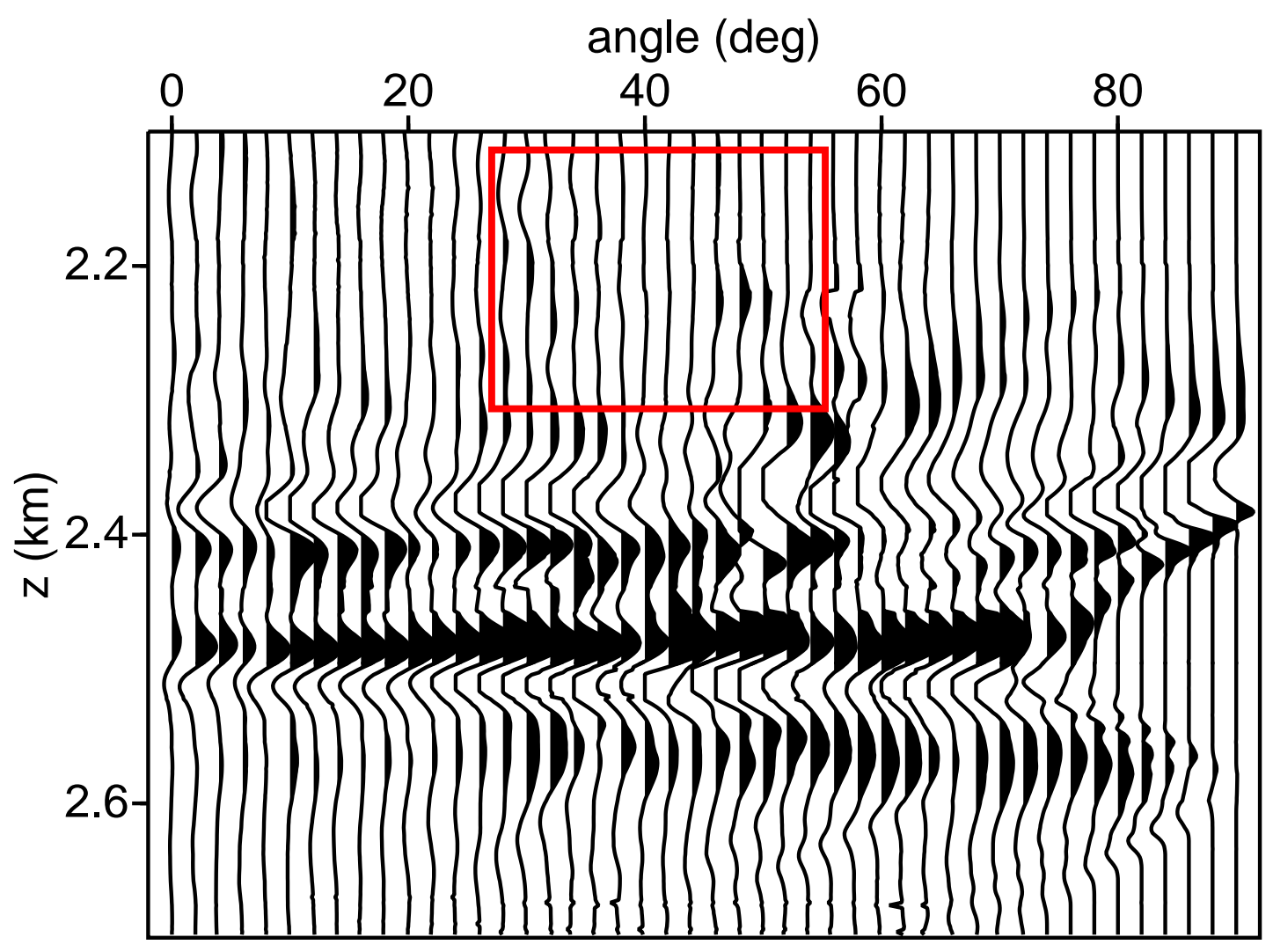

Authors: Stolk and Symes

Figure number : 18

File name(s) : marmim5withoutbox.eps

Column width

Color figure 


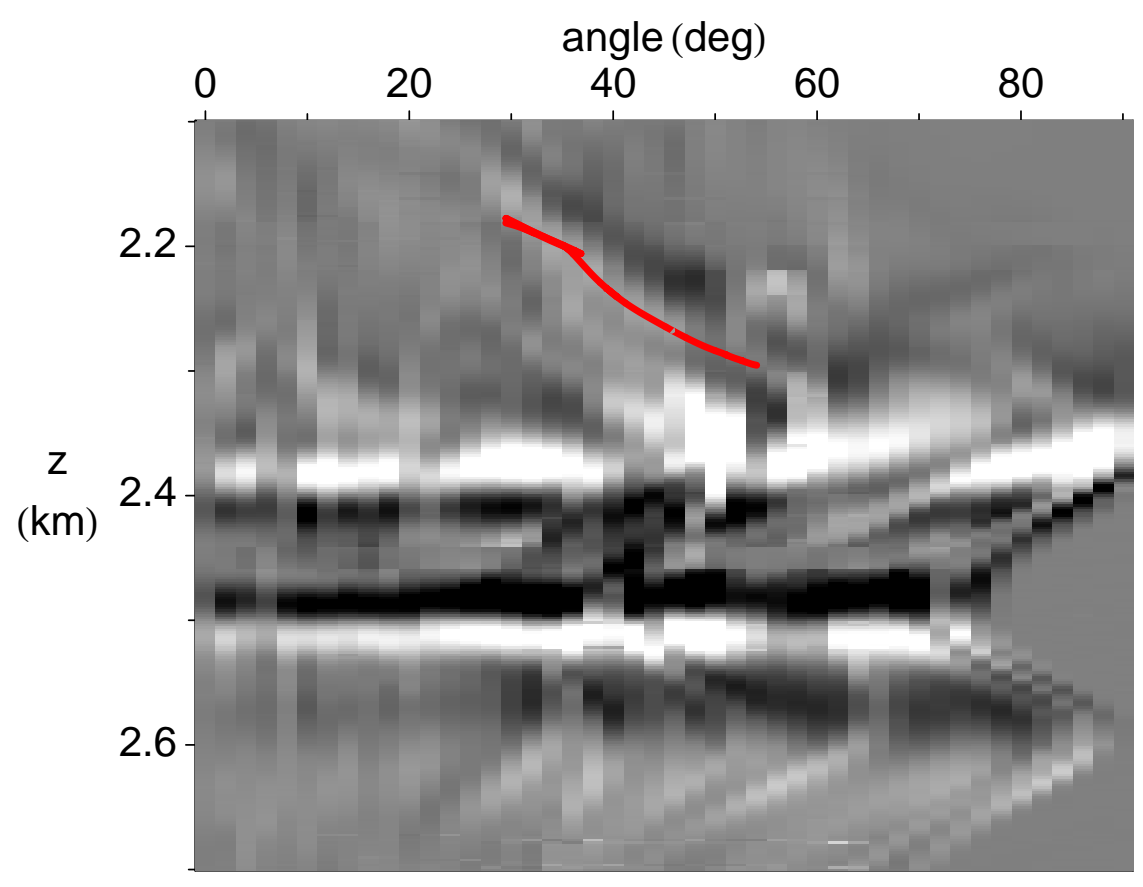

Authors: Stolk and Symes

Figure number : 19

File name(s) : marmim5artifact.eps

Column width

Color figure 


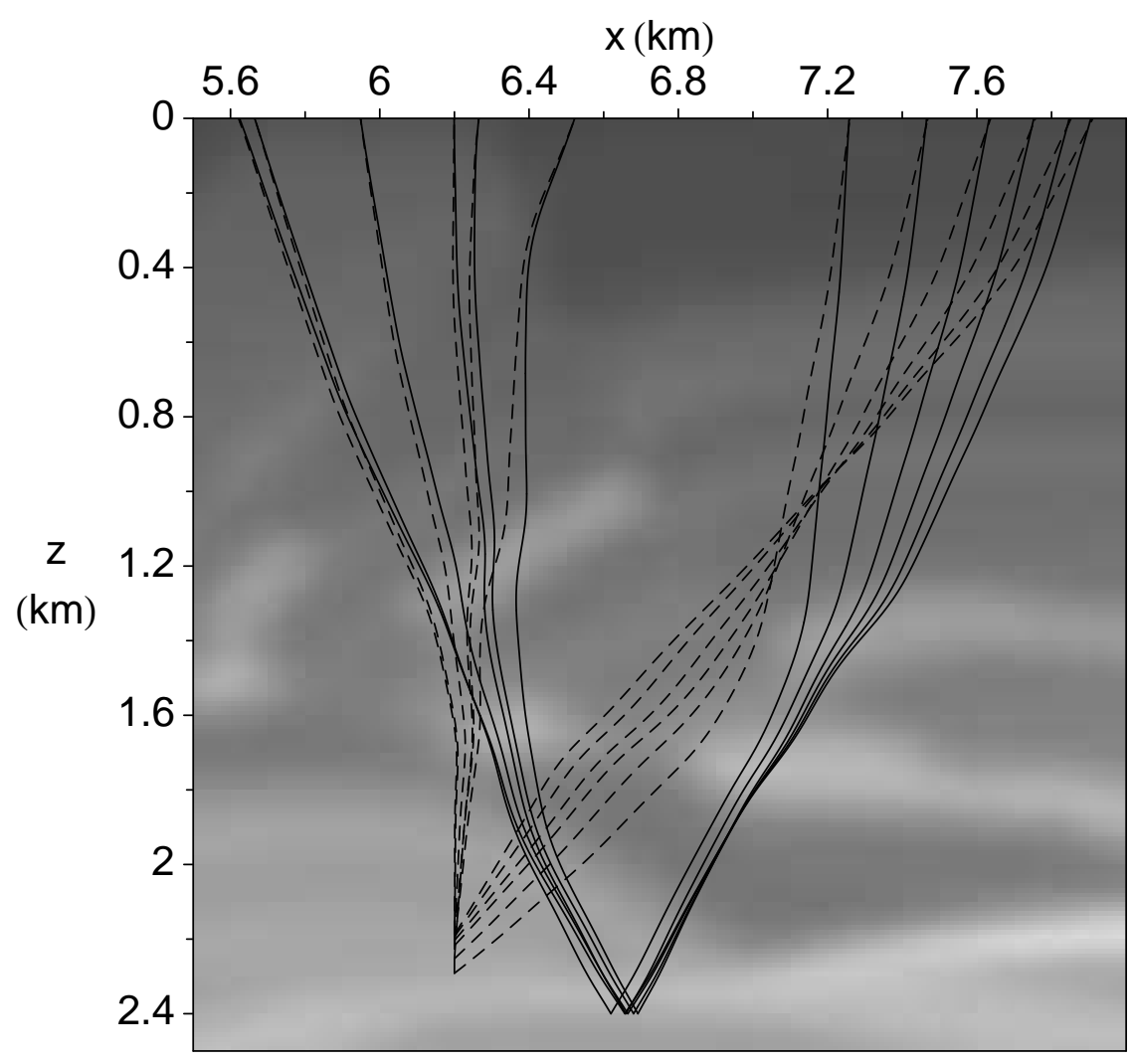

Authors: Stolk and Symes

Figure number : 20

File name(s): marmartifactraysnocolor.eps

Column width 